\title{
High-frequency sound wave propagation in binary gas mixtures flowing through microchannels
}

Cite as: Phys. Fluids 28, 052003 (2016); https://doi.org/10.1063/1.4948657

Submitted: 10 August 2015 . Accepted: 24 April 2016 . Published Online: 10 May 2016

M. Bisi (iD), and S. Lorenzani
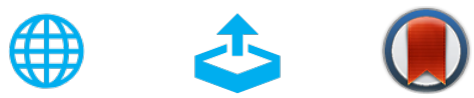

View Online

Export Citation

CrossMark

\section{ARTICLES YOU MAY BE INTERESTED IN}

Sound propagation through a rarefied gas confined between source and receptor at arbitrary Knudsen number and sound frequency

Physics of Fluids 21, 103601 (2009); https://doi.org/10.1063/1.3247159

Sound wave resonances in micro-electro-mechanical systems devices vibrating at high frequencies according to the kinetic theory of gases

Physics of Fluids 24, 092001 (2012); https://doi.org/10.1063/1.4748966

Growth of sinuous waves on thin liquid sheets: Comparison of predictions with experiments

Physics of Fluids 28, 052101 (2016); https://doi.org/10.1063/1.4948269

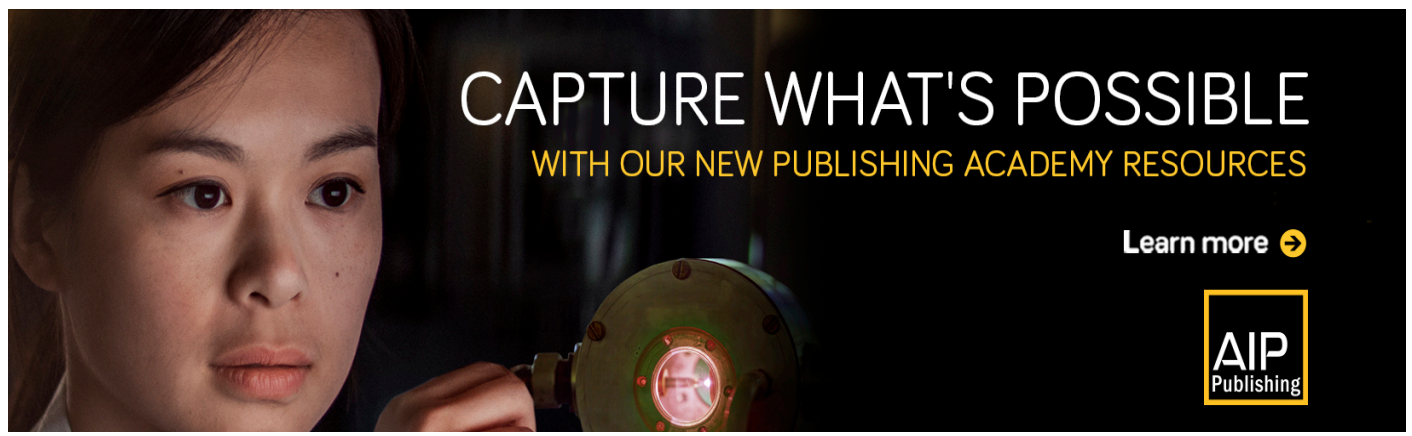




\title{
High-frequency sound wave propagation in binary gas mixtures flowing through microchannels
}

\author{
M. Bisi ${ }^{1}$ and S. Lorenzani ${ }^{2, a)}$ \\ ${ }^{1}$ Dipartimento di Matematica e Informatica, Universita' di Parma, Parco Area delle \\ Scienze 53/A, I-43124 Parma, Italy \\ ${ }^{2}$ Dipartimento di Matematica, Politecnico di Milano, Piazza Leonardo da Vinci 32, \\ I-20133 Milano, Italy
}

(Received 10 August 2015; accepted 24 April 2016; published online 10 May 2016)

\begin{abstract}
The propagation of high-frequency sound waves in binary gas mixtures flowing through microchannels is investigated by using the linearized Boltzmann equation based on a Bhatnagar-Gross-Krook (BGK)-type approach and diffuse reflection boundary conditions. The results presented refer to mixtures whose constituents have comparable molecular mass (like Ne-Ar) as well as to disparate-mass gas mixtures (composed of very heavy plus very light molecules, like $\mathrm{He}-\mathrm{Xe}$ ). The sound wave propagation model considered in the present paper allows to analyze the precise nature of the forced-sound modes excited in different gas mixtures. Published by AIP Publishing. [http://dx.doi.org/10.1063/1.4948657]
\end{abstract}

\section{INTRODUCTION}

Sound-wave propagation through a gas is known to be adequately described by the continuum Navier-Stokes equations, provided the characteristic length of the gas flow domain is significantly larger than the molecular mean free path. However, if the excitation frequency $\omega$ of the sound wave becomes sufficiently high, the classical continuum approach fails even at ordinary densities, because $\omega^{-1}$ can become of the order of the molecular mean free time. Traditional moment methods also fail at high frequencies. Methods akin to Grad's procedure were used by Wang Chang and Uhlenbeck ${ }^{1}$ and by Pekeris et al. ${ }^{2}$ in order to describe sound propagation, but the results for the attenuation rate were found in complete disagreement with experiments at high frequencies. ${ }^{3-5}$ Therefore, in the case of rarefied gas dynamics (or equivalently, in the case of a highly oscillatory phenomenon), there is no recourse but to turn to the kinetic theory and the Boltzmann equation. ${ }^{6-17}$ In the sixties, $\mathrm{Grad}^{18}$ conjectured that when a binary gas mixture is made up of species with very different molecular masses, under appropriate circumstances, the two components of such a mixture can support different temperatures, due to the slow exchange of kinetic energy between light and heavy species. Two-temperature continuum equations, necessary to replace those of hydrodynamics in part of the continuum regime, have been developed by a number of authors. ${ }^{19-29}$ From such "two-temperature hydrodynamics", Huck and Johnson ${ }^{30,31}$ predicted the possibility of several simultaneous sound modes in a disparate-mass gas mixture (composed of very heavy plus very light molecules), for a narrow range of compositions at high enough frequencies. However, the identity of these interfering modes has never been completely understood over the years. Thus, a deeper analysis of forced sound propagation in a gas mixture is expected to be particularly interesting, in order to clarify the long standing open question about the precise nature of sound modes excited in disparate-mass gas mixtures. ${ }^{30,32-35}$

Furthermore, a correct description of sound propagation through a rarefied gas is very important in connection with the evaluation of damping forces in micro-electro-mechanical system (MEMS) devices vibrating at high frequencies. In the last few years, high-frequency MEMS devices (RF MEMS) (ranging from $1 \mathrm{MHz}$ to $60 \mathrm{GHz}$ ) have increasingly been used in various industrial

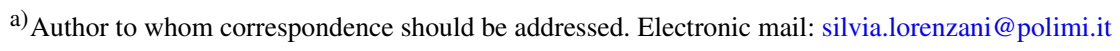


fields. Low frequency MEMS devices are normally operated at very low pressure in order to minimize the damping due to the internal friction of the gas (viscous damping). ${ }^{36}$ This need can be overcome when MEMS devices vibrate at relatively high frequencies, since gas compressibility and inertial forces lead then to another damping mechanism, which is related to the propagation of sound waves generated by high-frequency oscillating micro-structures. Very recently, it has been proved in Desvillettes and Lorenzani, ${ }^{37}$ for a single-component gas, that sound waves propagating between the micro-device walls induce a resonant/antiresonant response of the system. The occurrence of an antiresonance is particularly important since, if the device is operated close to the corresponding frequency, the damping due to the gas is considerably reduced. Since gas resonances take place for each value of the rarefaction parameter (inverse Knudsen number), the RF MEMS devices can perform well also at atmospheric pressure, greatly reducing the need for (and the cost associated with) vacuum packaging.

In the current investigation, we extend the previous analysis carried out for a single species gas $^{37}$ to a gas mixture. The kinetic description of a mixture of gases with different particle masses (and possibly with different internal energies) is not a trivial generalization of the classical Boltzmann theory for a single gas, since the collision operators have to take into account exchanges of momentum and energy among the different species (and also mass exchanges, in the case of reacting mixtures). ${ }^{24,25,28}$ In the present paper, we will use the unsteady kinetic equation based on the linearized version of the relaxation model of BGK-type presented in Groppi and Spiga ${ }^{38}$ This BGK approximation has already been used as a consistent tool to investigate other physical problems, such as condensation and sublimation in gas mixtures. ${ }^{39,40}$ The evaluation of damping forces exerted by gaseous mixtures, that we want to investigate here, assumes a basic relevance in all MEMS fabrication processes. In fact, during the wafer bonding process, a gas mixture of noble gases (like $\mathrm{Ar}, \mathrm{Kr}$, or Ne) and getterable gases (like $\mathrm{N}_{2}, \mathrm{O}_{2}$, or $\mathrm{CO}_{2}$ ) is usually backfilled into the MEMS sensor package to set its operating pressure, which should exactly match the damping requirements of the micro-device design ("backfilling process"). ${ }^{41}$ Beyond the analysis of damping forces in RF MEMS, the sound wave propagation model considered in the present paper allows us to study the nature of the multiple forced-sound modes excited in disparate-mass gas mixtures, thanks to the peculiar characteristics shown by the sound waves in correspondence of the resonant/antiresonant frequency.

\section{LINEARIZED BGK MODEL FOR A BINARY MIXTURE}

In kinetic theory, the evolution of a mixture of $N$ elastically scattering gases is usually described by a set of $N$ integro-differential equations of Boltzmann type for the species distribution functions $f^{s}(t, \mathbf{x}, \xi)(s=1, \ldots, N){ }^{42,43}$ Since it is difficult, in general, to manage the collision integral operator as such, simplified kinetic models have been developed in the literature ${ }^{44-47}$ and widely used in practice. ${ }^{48-52}$ In the present paper, we perform our analysis by using the BGK relaxation model proposed by Andries et al. ${ }^{53}$ and generalized by Groppi and Spiga ${ }^{38}$ even to non-conservative (reactive) collisions, which has been proven to be well posed from the mathematical point of view: correct Boltzmann collision invariants and Maxwellian equilibria are properly recovered, the $H$-theorem is fulfilled, and the indifferentiability principle holds (when the $N$ gases coincide, the classical BGK model for a single gas is correctly reproduced).

For a mixture of two gases $(N=2)$ with particle masses $m^{1}, m^{2}$, the BGK model presented by Groppi and $\mathrm{Spiga}^{38}$ reads as

$$
\frac{\partial f^{s}}{\partial t}+\xi \cdot \nabla_{\mathbf{x}} f^{s}=v_{s}\left(\mathcal{M}_{s}-f^{s}\right) \quad s=1,2,
$$

where $v_{s}$ are suitable collision frequencies (independent from the molecular velocity $\xi$, but possibly dependent on the macroscopic fields) and $\mathcal{M}_{s}$ are Maxwellian attractors,

$$
\mathcal{M}_{s}=n^{s}\left(\frac{m^{s}}{2 \pi k T_{s}}\right)^{3 / 2} \exp \left[-\frac{m^{s}}{2 k T_{s}}\left|\xi-\mathbf{v}_{s}\right|^{2}\right]
$$

with $k$ being the Boltzmann constant. Auxiliary parameters $\mathbf{v}_{s}, T_{s}$ (with subscript $s$ ) are determined in terms of the moments of the distribution functions $f^{s}$, which is the number density $n^{s}$, the 
mass velocity $\mathbf{v}^{s}$, the temperature $T^{s}$ (with superscript $s$ ), by imposing that the exchange rates for species momenta and energies given by the BGK operator reproduce the exact corresponding rates calculated by the Boltzmann collision operators $Q^{s r}\left(f^{s}, f^{r}\right)$,

$$
\begin{gathered}
v_{s} m^{s} \int_{\mathbb{R}} \xi\left[\mathcal{M}_{s}(\xi)-f^{s}(\xi)\right] d \xi=\sum_{r=1}^{2} m^{s} \int_{\mathbb{R}} \xi Q^{s r}\left(f^{s}, f^{r}\right) d \xi, \\
v_{s} \frac{m^{s}}{2} \int_{\mathbb{R}}|\xi|^{2}\left[\mathcal{M}_{s}(\xi)-f^{s}(\xi)\right] d \xi=\sum_{r=1}^{2} \frac{m^{s}}{2} \int_{\mathbb{R}}|\xi|^{2} Q^{s r}\left(f^{s}, f^{r}\right) d \xi,
\end{gathered}
$$

when collisions are of Maxwell molecule type. Skipping intermediate details, the final expressions of the auxiliary fields written in compact form (for $s=1,2$ ) are

$$
\begin{gathered}
\mathbf{v}_{s}=\mathbf{v}^{s}+(-1)^{s} \frac{v_{1}^{12}}{v_{s}} \frac{\mu^{12}}{m^{s}} \frac{n^{1} n^{2}}{n^{s}}\left(\mathbf{v}^{1}-\mathbf{v}^{2}\right), \\
k T_{s}=k T^{s}-(-1)^{s} \frac{2}{3} \frac{v_{1}^{12}}{v_{s}} \mu^{12} \frac{n^{1} n^{2}}{n^{s}} \mathbf{v}^{s} \cdot\left(\mathbf{v}^{1}-\mathbf{v}^{2}\right) \\
-\frac{1}{3 m^{s}}\left(\frac{v_{1}^{12}}{v_{s}} \mu^{12} \frac{n^{1} n^{2}}{n^{s}}\right)^{2}\left|\mathbf{v}^{1}-\mathbf{v}^{2}\right|^{2}+2(-1)^{s} \frac{v_{1}^{12}}{v_{s}} \frac{\mu^{12}}{\left(m^{1}+m^{2}\right)} \frac{n^{1} n^{2}}{n^{s}} k\left(T^{1}-T^{2}\right) \\
+\frac{2}{3}(-1)^{s} \frac{v_{1}^{12}}{v_{s}} \frac{\mu^{12}}{\left(m^{1}+m^{2}\right)} \frac{n^{1} n^{2}}{n^{s}}\left(m^{1} \mathbf{v}^{1}+m^{2} \mathbf{v}^{2}\right) \cdot\left(\mathbf{v}^{1}-\mathbf{v}^{2}\right),
\end{gathered}
$$

where $\mu^{12}=m^{1} m^{2} /\left(m^{1}+m^{2}\right)$ stands for the reduced mass, and $v_{k}^{s r}$ are suitably weighted integrals of the differential cross sections relevant to collisions between particles of species $s$ and $r$. Values for $v_{k}^{s r}$ corresponding to intermolecular potentials of inverse power type are reported in Chapman and Cowling. ${ }^{42}$ For the special case of the Maxwell molecule interparticle force law

$$
F^{s r}=\frac{K^{s r}}{r_{d}^{5}}
$$

(where $r_{d}$ is the distance between particles and $K^{s r}$ is the interparticle force law constant), the microscopic collision frequencies, relevant in the present analysis for a binary mixture, are explicitly given by

$$
\begin{aligned}
v_{1}^{12} & =2 \pi A_{1}(5)\left(\frac{K^{12}}{\mu^{12}}\right)^{1 / 2}, & v_{2}^{12} & =2 \pi A_{2}(5)\left(\frac{K^{12}}{\mu^{12}}\right)^{1 / 2}, \\
v_{2}^{11} & =2 \pi A_{2}(5)\left(\frac{2 K^{11}}{m^{1}}\right)^{1 / 2}, & v_{2}^{22} & =2 \pi A_{2}(5)\left(\frac{2 K^{22}}{m^{2}}\right)^{1 / 2} .
\end{aligned}
$$

Here $A_{1}(5) \simeq 0.422, A_{2}(5) \simeq 0.436$ are the dimensionless collision cross sections, ${ }^{42}$ while $K^{11}$ and $K^{22}$ can be written in terms of the simple gas viscosity coefficients $\eta^{s}(s=1,2)$ with aid of the first Chapman-Enskog expressions for these quantities,

$$
\eta^{s}=\frac{1}{3 \pi}\left(\frac{2 m^{s}}{K^{s s}}\right)^{1 / 2} \frac{(k T)}{A_{2}(5)}
$$

and the constant $K^{12}$ may be determined from the method of the combination rule

$$
K^{12}=\left(K^{11} K^{22}\right)^{1 / 2}
$$

previously considered by McCormack. ${ }^{44}$ In order to specify the force constants $K^{11}$ and $K^{22}$, use has been made of the experimental data on the viscosities $\eta^{s}$ of the single gases at the temperature $T=300 \mathrm{~K}$, given in Kestin et al. ${ }^{54}$

A useful tool in the sequel will be the linearization of the BGK equations (1), obtained representing the distribution functions $f^{s}$ of both species as follows:

$$
f^{s}=f_{0}^{s}\left(1+h^{s}\right), \quad\left|h^{s}\right| \ll 1,
$$


with $f_{0}^{s}$ being the Maxwellian configuration

$$
f_{0}^{s}=n_{0}^{s}\left(\frac{m^{s}}{2 \pi k T_{0}}\right)^{3 / 2} \exp \left[-\frac{m^{s}}{2 k T_{0}} \xi^{2}\right]
$$

and $h^{s}$ being the small perturbation with respect to the equilibrium state. Neglecting higher order terms, it can be easily checked that

$$
\begin{gathered}
n^{s}=n_{0}^{s}+\int_{\mathbf{R}^{3}} f_{0}^{s} h^{s} d \xi, \\
\mathbf{v}^{s}=\frac{1}{n_{0}^{s}} \int_{\mathbf{R}^{3}} \xi f_{0}^{s} h^{s} d \xi, \\
T^{s}=T_{0}+\frac{m^{s}}{3 k n_{0}^{s}} \int_{\mathbf{R}^{3}}\left(|\xi|^{2}-\frac{3 k}{m^{s}} T_{0}\right) f_{0}^{s} h^{s} d \xi, \\
P_{i j}^{s}=P_{0}^{s} \delta_{i j}+\int_{\mathbf{R}^{3}} \xi_{i} \xi_{j} f_{0}^{s} h^{s} d \xi,
\end{gathered}
$$

where $P_{i j}^{s}$ denotes the viscous stress tensor. We omit here all detailed computations leading to the linearized BGK operator, since we follow exactly the same steps of the linearization of classical BGK model for a single gas presented in Cercignani. ${ }^{43}$ From now on, we introduce the normalizations

$$
\begin{gathered}
\mathbf{c}=\frac{\xi}{\sqrt{2 \frac{k}{m^{1}} T_{0}}}, \\
\hat{f}_{0}^{1}=\frac{f_{0}^{1}}{n_{0}^{1}}=\frac{\mathrm{e}^{-|\mathbf{c}|^{2}}}{\pi^{3 / 2}}, \\
\hat{f}_{0}^{2}=\frac{f_{0}^{2}}{n_{0}^{2}}=\frac{\mathrm{e}^{-\frac{|\mathbf{c}|^{2}}{M_{12}}}}{\left(\pi M_{12}\right)^{3 / 2}},
\end{gathered}
$$

where $M_{12}=m^{1} / m^{2}$ is the mass ratio. The unusual choice of normalizing the molecular velocities with respect to the thermal velocity of the species 1 depends on the fact that, in this way, it becomes more evident to identify in the equations written for the components of the mixture (and reported in Sec. III) the deviations from the single gas behavior, in terms of peculiar parameters such as the molecular mass ratio, $M_{12}$, and the macroscopic collision frequencies ratio, $\Theta_{12}=v_{2} / v_{1}$. In particular, the mass ratio parameter $M_{12}$ will be useful in the sequel to identify the cases of mixture with comparable particle masses $\left(M_{12} \rightarrow 1\right)$ or with disparate masses $\left(M_{12} \rightarrow 0\right.$, if we assume $\left.m_{1}<m_{2}\right)$. Consequently, the linearized version of the BGK collision term, given by the right-hand side of Eq. (1), can be written as

$$
\begin{gathered}
L h_{B G K}^{1}=v_{1}\left\{\rho^{1}+2\left(1-\Gamma^{1}\right) \mathbf{c} \cdot \mathbf{v}^{1}+2 \Gamma^{1} \mathbf{c} \cdot \mathbf{v}^{2}+\left[1-\frac{2 \Gamma^{1} M_{12}}{\left(1+M_{12}\right)}\right]\left(|\mathbf{c}|^{2}-\frac{3}{2}\right) \tau^{1}\right. \\
\left.+\frac{2 \Gamma^{1} M_{12}}{\left(1+M_{12}\right)}\left(|\mathbf{c}|^{2}-\frac{3}{2}\right) \tau^{2}-h^{1}\right\}, \\
L h_{B G K}^{2}=v_{2}\left\{\rho^{2}+2\left(1-\Gamma^{2}\right) \frac{\mathbf{c}}{M_{12}} \cdot \mathbf{v}^{2}+2 \Gamma^{2} \frac{\mathbf{c}}{M_{12}} \cdot \mathbf{v}^{1}+\left[1-\frac{2 \Gamma^{2}}{\left(1+M_{12}\right)}\right]\left(\frac{|\mathbf{c}|^{2}}{M_{12}}-\frac{3}{2}\right) \tau^{2}\right. \\
\left.+\frac{2 \Gamma^{2}}{\left(1+M_{12}\right)}\left(\frac{|\mathbf{c}|^{2}}{M_{12}}-\frac{3}{2}\right) \tau^{1}-h^{2}\right\},
\end{gathered}
$$

where

$$
\Gamma^{1}=\frac{v_{1}^{12} \mu^{12}}{v_{1} m^{1}} n_{0}^{2}, \quad \Gamma^{2}=\frac{v_{1}^{12} \mu^{12}}{v_{2} m^{2}} n_{0}^{1},
$$

while the dimensionless macroscopic perturbed density $\rho^{s}$, velocity $\mathbf{v}^{s}$, and temperature $\tau^{s}$ are defined as 


$$
\begin{array}{rlrl}
\rho^{1} & =\frac{1}{\pi^{3 / 2}} \int_{\mathbb{R}^{3}} h^{1} \mathrm{e}^{-|\mathbf{c}|^{2}} d \mathbf{c}, & \rho^{2}=\frac{1}{\left(\pi M_{12}\right)^{3 / 2}} \int_{\mathbb{R}^{3}} h^{2} \mathrm{e}^{-\frac{|\mathbf{c}|^{2}}{M_{12}}} d \mathbf{c}, \\
\mathbf{v}^{1}=\frac{1}{\pi^{3 / 2}} \int_{\mathbb{R}^{3}} \mathbf{c} h^{1} \mathrm{e}^{-|\mathbf{c}|^{2}} d \mathbf{c}, & \mathbf{v}^{2}=\frac{1}{\left(\pi M_{12}\right)^{3 / 2}} \int_{\mathbb{R}^{3}} \mathbf{c} h^{2} \mathrm{e}^{-\frac{|\mathbf{c}|^{2}}{M_{12}}} d \mathbf{c}, \\
\tau^{1}=\frac{1}{\pi^{3 / 2}} \int_{\mathbb{R}^{3}}\left(\frac{2}{3}|\mathbf{c}|^{2}-1\right) h^{1} \mathrm{e}^{-|\mathbf{c}|^{2}} d \mathbf{c}, & \tau^{2}=\frac{1}{\left(\pi M_{12}\right)^{3 / 2}} \int_{\mathbb{R}^{3}}\left(\frac{2|\mathbf{c}|^{2}}{3 M_{12}}-1\right) h^{2} \mathrm{e}^{-\frac{|\mathbf{c}|^{2}}{M_{12}}} d \mathbf{c} .
\end{array}
$$

Crucial properties of this BGK model (preservation of correct collision equilibria, conservation laws, and $H$-theorem) hold independently from the choice of collision frequencies $v_{1}, v_{2}$. In the present paper, we determine $v_{1}, v_{2}$ by imposing, as additional constraint, that the (linearized) BGK model reproduces the (linearized) exchange rates for viscous stress tensors $P_{i j}^{1}$ and $P_{i j}^{2}$ (defined in (12)) prescribed by the Boltzmann equations, but other consistent options could be adopted for them. ${ }^{25}$ Skipping calculation details, such a constraint yields

$$
\begin{aligned}
& v_{1}=\frac{3}{4} v_{2}^{11} n_{0}^{1}+\frac{3}{2} v_{2}^{12} \frac{1}{1+M_{12}} n_{0}^{2}, \\
& v_{2}=\frac{3}{2} v_{2}^{12} \frac{M_{12}}{1+M_{12}} n_{0}^{1}+\frac{3}{4} v_{2}^{22} n_{0}^{2} .
\end{aligned}
$$

\section{SOUND WAVE PROPAGATION MODEL}

Let us consider a binary gaseous mixture confined between two flat, infinite, and parallel plates located at $z^{\prime}=-d / 2$ and $z^{\prime}=d / 2$. Both boundaries are held at the same constant temperature. The upper wall of the channel (located at $z^{\prime}=d / 2$ ) is fixed while the lower one (located at $z^{\prime}=-d / 2$ ) harmonically oscillates in the $z^{\prime}$-direction (normal to the wall itself) with angular frequency $\omega^{\prime}$ (the corresponding period being $\left.T^{\prime}=2 \pi / \omega^{\prime}\right)$. The velocity $U_{w}^{\prime}$ of the oscillating plate depends on time $t^{\prime}$ through the formula

$$
U_{w}^{\prime}\left(t^{\prime}\right)=U_{0}^{\prime} \sin \left(\omega^{\prime} t^{\prime}\right),
$$

where it is assumed that the amplitude $U_{0}^{\prime}$ is very small compared to the characteristic molecular velocity of the mixture given by

$$
v_{0}=\sqrt{2 k T_{0} / m},
$$

with $m$ being the mean molecular mass of the mixture and $T_{0}$ being the equilibrium temperature of the mixture. Under these conditions, the Boltzmann equation modeling the gaseous mixture motion inside the channel can be linearized, as described in Sec. II. Using Eq. (7), the system of nonstationary BGK-Boltzmann equations reads as

$$
\frac{\partial h^{s}}{\partial t^{\prime}}+c_{z} \frac{\partial h^{s}}{\partial z^{\prime}}=L h_{B G K}^{s} \quad s=1,2
$$

where $L h_{B G K}^{s}$ is the linearized BGK collision operator given by Eqs. (16)-(17). It is convenient now to rescale all variables appearing in Eq. (22) as follows:

$$
t=t^{\prime} / \theta_{1}, \quad z=z^{\prime} /\left(v_{0}^{1} \theta_{1}\right),
$$

with $\theta_{1}=1 / v_{1}, v_{0}^{1}=\sqrt{2 k T_{0} / m^{1}}$. Furthermore, we define $\Theta_{12}=\theta_{1} / \theta_{2}=v_{2} / v_{1}$ and $\delta=d /\left(v_{0}^{1} \theta_{1}\right)$, which is the dimensionless distance between the channel walls as well as the rarefaction parameter (inverse Knudsen number) of the species $s=1$. Since the problem under consideration is one-dimensional in space, the unknown perturbed distribution functions $h^{s}$, as well as the overall quantities, depend only on the $z$ coordinate. Likewise, we can reduce the dimensionality of the molecular-velocity space by introducing the projection procedure. ${ }^{17,55,56}$ First, we multiply Eq. (22) by $\frac{1}{\pi} e^{-\left(c_{x}^{2}+c_{y}^{2}\right)}$, when $s=1$, and by $\frac{1}{\left(\pi M_{12}\right)} e^{-\left(c_{x}^{2}+c_{y}^{2}\right) / M_{12}}$, when $s=2$, and we integrate over all $c_{x}$ and $c_{y}$. Then, we multiply Eq. (22) by $\frac{1}{\pi}\left(c_{x}^{2}+c_{y}^{2}-1\right) e^{-\left(c_{x}^{2}+c_{y}^{2}\right)}$, when $s=1$, and by 
$\frac{1}{\left(\pi M_{12}\right)}\left(\frac{c_{x}^{2}+c_{y}^{2}}{M_{12}}-1\right) e^{-\left(c_{x}^{2}+c_{y}^{2}\right) / M_{12}}$, when $s=2$, and we integrate again over all $c_{x}$ and $c_{y}$. The resulting equations after the projection are

$$
\begin{gathered}
\frac{\partial H^{1}}{\partial t}+c_{z} \frac{\partial H^{1}}{\partial z}+H^{1}=\rho^{1}+2\left(1-\Gamma^{1}\right) c_{z} v_{z}^{1}+2 \Gamma^{1} c_{z} v_{z}^{2}+\left[1-\frac{2 \Gamma^{1} M_{12}}{\left(1+M_{12}\right)}\right]\left(c_{z}^{2}-\frac{1}{2}\right) \tau^{1} \\
+\frac{2 \Gamma^{1} M_{12}}{\left(1+M_{12}\right)}\left(c_{z}^{2}-\frac{1}{2}\right) \tau^{2}, \\
\frac{\partial H^{2}}{\partial t}+c_{z} \frac{\partial H^{2}}{\partial z}+\Theta_{12} H^{2}=\Theta_{12}\left\{\rho^{2}+\frac{2\left(1-\Gamma^{2}\right)}{M_{12}} c_{z} v_{z}^{2}\right. \\
\left.+\frac{2 \Gamma^{2}}{M_{12}} c_{z} v_{z}^{1}+\left[1-\frac{2 \Gamma^{2}}{\left(1+M_{12}\right)}\right]\left(\frac{c_{z}^{2}}{M_{12}}-\frac{1}{2}\right) \tau^{2}+\frac{2 \Gamma^{2}}{\left(1+M_{12}\right)}\left(\frac{c_{z}^{2}}{M_{12}}-\frac{1}{2}\right) \tau^{1}\right\}, \\
\frac{\partial \Psi^{1}}{\partial t}+c_{z} \frac{\partial \Psi^{1}}{\partial z}+\Psi^{1}=\left[1-\frac{2 \Gamma^{1} M_{12}}{\left(1+M_{12}\right)}\right] \tau^{1}+\frac{2 \Gamma^{1} M_{12}}{\left(1+M_{12}\right)} \tau^{2}, \\
\frac{\partial \Psi^{2}}{\partial t}+c_{z} \frac{\partial \Psi^{2}}{\partial z}+\Theta_{12} \Psi^{2}=\Theta_{12}\left\{\left[1-\frac{2 \Gamma^{2}}{\left(1+M_{12}\right)}\right] \tau^{2}+\frac{2 \Gamma^{2}}{\left(1+M_{12}\right)} \tau^{1}\right\},
\end{gathered}
$$

where the reduced unknown distribution functions $H^{s}$ and $\Psi^{s}$ are defined as

$$
\begin{gathered}
H^{1}\left(z, c_{z}, t\right)=\frac{1}{\pi} \int_{-\infty}^{+\infty} \int_{-\infty}^{+\infty} h^{1}(z, \mathbf{c}, t) e^{-\left(c_{x}^{2}+c_{y}^{2}\right)} d c_{x} d c_{y}, \\
H^{2}\left(z, c_{z}, t\right)=\frac{1}{\pi M_{12}} \int_{-\infty}^{+\infty} \int_{-\infty}^{+\infty} h^{2}(z, \mathbf{c}, t) e^{-\left(c_{x}^{2}+c_{y}^{2}\right) / M_{12}} d c_{x} d c_{y}, \\
\Psi^{1}\left(z, c_{z}, t\right)=\frac{1}{\pi} \int_{-\infty}^{+\infty} \int_{-\infty}^{+\infty}\left(c_{x}^{2}+c_{y}^{2}-1\right) h^{1}(z, \mathbf{c}, t) e^{-\left(c_{x}^{2}+c_{y}^{2}\right)} d c_{x} d c_{y}, \\
\Psi^{2}\left(z, c_{z}, t\right)=\frac{1}{\pi M_{12}} \int_{-\infty}^{+\infty} \int_{-\infty}^{+\infty}\left(\frac{c_{x}^{2}+c_{y}^{2}}{M_{12}}-1\right) h^{2}(z, \mathbf{c}, t) e^{-\left(c_{x}^{2}+c_{y}^{2}\right) / M_{12}} d c_{x} d c_{y} .
\end{gathered}
$$

The macroscopic fields appearing on the right-hand side of Eqs. (24)-(27) are given by

$$
\begin{gathered}
\rho^{1}(z, t)=\frac{1}{\sqrt{\pi}} \int_{-\infty}^{+\infty} H^{1} e^{-c_{z}^{2}} d c_{z}, \\
\rho^{2}(z, t)=\frac{1}{\sqrt{\pi M_{12}}} \int_{-\infty}^{+\infty} H^{2} e^{-c_{z}^{2} / M_{12}} d c_{z}, \\
v_{z}^{1}(z, t)=\frac{1}{\sqrt{\pi}} \int_{-\infty}^{+\infty} c_{z} H^{1} e^{-c_{z}^{2}} d c_{z}, \\
v_{z}^{2}(z, t)=\frac{1}{\sqrt{\pi M_{12}}} \int_{-\infty}^{+\infty} c_{z} H^{2} e^{-c_{z}^{2} / M_{12}} d c_{z}, \\
\tau^{1}(z, t)=\frac{1}{\sqrt{\pi}} \int_{-\infty}^{+\infty} \frac{2}{3}\left[\left(c_{z}^{2}-1 / 2\right) H^{1}+\Psi^{1}\right] e^{-c_{z}^{2}} d c_{z}, \\
\frac{1}{\sqrt{\pi M_{12}}} \int_{-\infty}^{+\infty} \frac{2}{3}\left[\left(\frac{c_{z}^{2}}{M_{12}}-\frac{1}{2}\right) H^{2}+\Psi^{2}\right] e^{-c_{z}^{2} / M_{12}} d c_{z} .
\end{gathered}
$$

Applying the same projection procedure to the linearized boundary conditions reported in Desvillettes and Lorenzani ${ }^{37}$ (Eqs. (17)-(18)) describing the diffuse scattering of gaseous particles on both walls of the microchannel, we are led to derive the following expressions:

$$
\begin{aligned}
H^{1}\left(z=-\delta / 2, c_{z}, t\right)= & \left(\sqrt{\pi}+2 c_{z}\right) U_{w} \\
& -2 \int_{\tilde{c}_{z}<0} d \tilde{c}_{z} \tilde{c}_{z} e^{-\tilde{c}_{z}^{2}} H^{1}\left(z=-\delta / 2, \tilde{c}_{z}, t\right) \quad c_{z}>0,
\end{aligned}
$$




$$
\begin{array}{rlrl}
H^{2}\left(z=-\delta / 2, c_{z}, t\right)= & \left(\sqrt{\frac{\pi}{M_{12}}}+\frac{2 c_{z}}{M_{12}}\right) U_{w} & \\
& -\frac{2}{M_{12}} \int_{\tilde{c}_{z}<0} d \tilde{c}_{z} \tilde{c}_{z} e^{-\tilde{c}_{z}^{2} / M_{12}} H^{2}\left(z=-\delta / 2, \tilde{c}_{z}, t\right) & c_{z}>0, \\
\Psi^{1}\left(z=-\delta / 2, c_{z}, t\right)= & \Psi^{2}\left(z=-\delta / 2, c_{z}, t\right)=0 & c_{z}>0, \\
H^{1}\left(z=\delta / 2, c_{z}, t\right)= & 2 \int_{\tilde{c}_{z}>0} d \tilde{c}_{z} \tilde{c}_{z} e^{-\tilde{c}_{z}^{2}} H^{1}\left(z=\delta / 2, \tilde{c}_{z}, t\right) & c_{z}<0, \\
H^{2}\left(z=\delta / 2, c_{z}, t\right)=\frac{2}{M_{12}} \int_{\tilde{c}_{z}>0} d \tilde{c}_{z} \tilde{c}_{z} e^{-\tilde{c}_{z}^{2} / M_{12} H^{2}\left(z=\delta / 2, \tilde{c}_{z}, t\right)} & & c_{z}<0, \\
\Psi^{1}\left(z=\delta / 2, c_{z}, t\right)=\Psi^{2}\left(z=\delta / 2, c_{z}, t\right)=0 & c_{z}<0 .
\end{array}
$$

In Eqs. (38) and (39), $U_{w}$ is the dimensionless wall velocity given by

$$
U_{w}(t)=U_{0} \sin (\omega t)
$$

with $U_{w}=U_{w}^{\prime} / v_{0}^{1}, U_{0}=U_{0}^{\prime} / v_{0}^{1}, \omega=\theta_{1} \omega^{\prime}, T=2 \pi / \omega=T^{\prime} / \theta_{1}$. The time-dependent problem described by Eqs. (24)-(27), with boundary conditions given by Eqs. (38)-(43), has been numerically solved by a deterministic finite-difference method. ${ }^{57,58}$

In order to investigate more deeply the influence of the parameters peculiar to a binary gaseous mixture, such as the molecular mass ratio, $M_{12}$, and the macroscopic collision frequencies ratio, $\Theta_{12}$, on the sound wave propagation, it is convenient to derive a system of integral equations for the macroscopic fields of the two species. In particular, in view of our subsequent considerations, we are interested in writing down the integral equations for the bulk velocities of the gas components. Since the vibrations of the lower wall of the microchannel are generated by a time-harmonic forcing (of frequency $\omega$ ) of the form $\sin (\omega t)$ (see Eq. (44)), we introduce the following expression $U_{w}=U_{0} e^{i \omega t}$ in Eqs. (38) and (39) and then we look for solutions of Eqs. (24)-(27) under the form

$$
\begin{aligned}
& H^{s}\left(z, c_{z}, t\right)=\mathcal{H}^{s}\left(z, c_{z}\right) e^{i \omega t} \quad s=1,2, \\
& \Psi^{s}\left(z, c_{z}, t\right)=\Psi^{s}\left(z, c_{z}\right) e^{i \omega t} \quad s=1,2 .
\end{aligned}
$$

The solutions of the original problem are then recovered by taking the imaginary part of $H^{s}$ and $\Psi^{s}$. Inserting (45) and (46) in (24)-(27) and then integrating the resulting equations along the trajectories of the molecules, we obtain an explicit expression for the distribution functions $\mathcal{H}^{s}$ and $\Psi^{s}$, which should be inserted in the definitions (32)-(37). Since these calculations are an extension of those reported in Desvillettes and Lorenzani ${ }^{37}$ for a single component gas, we will skip all the details. Thus, the integral equations for the bulk velocities of the gas components read as follows:

$$
\begin{gathered}
\mathrm{v}_{z}^{1}(z)=\mathcal{K}_{1}(z, \gamma)+\frac{1}{\sqrt{\pi}} \int_{-\delta / 2}^{\delta / 2} d s \varrho^{1}(s) \operatorname{sgn}(z-s) T_{0}(|z-s| \gamma) \\
-\frac{1}{\sqrt{\pi}} \int_{-\delta / 2}^{\delta / 2} d s \varrho^{1}(s) \mathcal{K}_{2}(z, s, \gamma) \\
+\frac{2}{\sqrt{\pi}} \int_{-\delta / 2}^{\delta / 2} d s\left[\left(1-\Gamma^{1}\right) \mathrm{v}_{z}^{1}(s)+\Gamma^{1} \mathrm{v}_{z}^{2}(s)\right] T_{1}(|z-s| \gamma) \\
-\frac{1}{\sqrt{\pi}} \int_{-\delta / 2}^{\delta / 2} d s\left[\left(1-\Gamma^{1}\right) \mathrm{v}_{z}^{1}(s)+\Gamma^{1} \mathrm{v}_{z}^{2}(s)\right] \mathcal{K}_{3}(z, s, \gamma) \\
+\frac{1}{\sqrt{\pi}} \int_{-\delta / 2}^{\delta / 2} d s\left[\left(1-\frac{2 \Gamma^{1} M_{12}}{\left(1+M_{12}\right)}\right) \tau^{1}(s)+\frac{2 \Gamma^{1} M_{12}}{\left(1+M_{12}\right)} \tau^{2}(s)\right] \\
\times \operatorname{sgn}(z-s)\left[T_{2}(|z-s| \gamma)-\frac{1}{2} T_{0}(|z-s| \gamma)\right] \\
-\frac{4}{\sqrt{\pi}} \int_{-\delta / 2}^{\delta / 2} d s\left[\left(1-\frac{2 \Gamma^{1} M_{12}}{\left(1+M_{12}\right)}\right) \tau^{1}(s)+\frac{2 \Gamma^{1} M_{12}}{\left(1+M_{12}\right)} \tau^{2}(s)\right] \mathcal{K}_{4}(z, s, \gamma),
\end{gathered}
$$




$$
\begin{aligned}
& \mathrm{v}_{z}^{2}(z)=\mathcal{K}_{1}(z, \tilde{\gamma})+\frac{\Theta_{12}}{\sqrt{\pi}} \int_{-\delta / 2}^{\delta / 2} d s \varrho^{2}(s) \operatorname{sgn}(z-s) T_{0}(|z-s| \tilde{\gamma}) \\
& -\frac{\Theta_{12}}{\sqrt{\pi}} \int_{-\delta / 2}^{\delta / 2} d s \varrho^{2}(s) \mathcal{K}_{2}(z, s, \tilde{\gamma}) \\
& +\frac{2 \Theta_{12}}{\sqrt{\pi M_{12}}} \int_{-\delta / 2}^{\delta / 2} d s\left[\left(1-\Gamma^{2}\right) \mathrm{v}_{z}^{2}(s)+\Gamma^{2} \mathrm{v}_{z}^{1}(s)\right] T_{1}(|z-s| \tilde{\gamma}) \\
& -\frac{\Theta_{12}}{\sqrt{\pi M_{12}}} \int_{-\delta / 2}^{\delta / 2} d s\left[\left(1-\Gamma^{2}\right) \mathrm{v}_{z}^{2}(s)+\Gamma^{2} \mathrm{v}_{z}^{1}(s)\right] \mathcal{K}_{3}(z, s, \tilde{\gamma}) \\
& +\frac{\Theta_{12}}{\sqrt{\pi}} \int_{-\delta / 2}^{\delta / 2} d s\left[\left(1-\frac{2 \Gamma^{2}}{\left(1+M_{12}\right)}\right) \tau^{2}(s)+\frac{2 \Gamma^{2}}{\left(1+M_{12}\right)} \tau^{1}(s)\right] \\
& \times \operatorname{sgn}(z-s)\left[T_{2}(|z-s| \tilde{\gamma})-\frac{1}{2} T_{0}(|z-s| \tilde{\gamma})\right] \\
& -\frac{4 \Theta_{12}}{\sqrt{\pi}} \int_{-\delta / 2}^{\delta / 2} d s\left[\left(1-\frac{2 \Gamma^{2}}{\left(1+M_{12}\right)}\right) \tau^{2}(s)+\frac{2 \Gamma^{2}}{\left(1+M_{12}\right)} \tau^{1}(s)\right] \mathcal{K}_{4}(z, s, \tilde{\gamma}),
\end{aligned}
$$

where $\gamma=(1+i \omega), \tilde{\gamma}=\left(\Theta_{12}+i \omega\right) / \sqrt{M_{12}}$, and the symbols $\mathcal{K}_{i}(i=1, \ldots, 4)$ stand for expressions involving products of the Abramowitz functions $T_{n}$ defined by

$$
T_{n}(x):=\int_{0}^{+\infty} s^{n} e^{-s^{2}-x / s} d s .
$$

The explicit form of the functions $\mathcal{K}_{i}$ is given in Appendix A. A comparison of the integral equations (47)-(48) with the bulk velocity field obtained in Desvillettes and Lorenzani ${ }^{37}$ for a single gas (Eq. (67)) allows us to infer under what conditions the sound modes associated to the components of the mixture reduce to a single gas-based sound mode.

Beyond the macroscopic fields given by Eqs. (32)-(37), a further quantity of interest in the present problem is the perturbation of the global normal stress $P_{z z}$ (this is the quantity that is measured experimentally) evaluated at $z=-\delta / 2$, since it gives the force exerted by the gaseous mixture on the moving wall of the channel. In the frame of our linearized analysis, the normal component of the stress tensor of the mixture is defined in terms of the single component parameters as $P_{z z}=P_{z z}^{1}+P_{z z}^{2}$, where

$$
\begin{gathered}
P_{z z}^{1}(z, t)=\frac{1}{\sqrt{\pi}} \int_{-\infty}^{+\infty} c_{z}^{2} H^{1} e^{-c_{z}^{2}} d c_{z}, \\
P_{z z}^{2}(z, t)=\frac{1}{\sqrt{\pi M_{12}}} \int_{-\infty}^{+\infty} c_{z}^{2} H^{2} e^{-c_{z}^{2} / M_{12}} d c_{z} .
\end{gathered}
$$

The normal stress time-dependence is of the following known form:

$$
\left|P_{z z}\right| \sin (\omega t+\phi),
$$

where $\left|P_{z z}\right|$ is the amplitude and $\phi$ the phase. In general, the amplitude of the time-dependent macroscopic fields is extracted from our numerical results as half the vertical distance between a maximum and the nearest minimum appearing in the temporal evolution of the macroscopic quantity. Since the parameter range investigated in our numerical simulations is close to the one taken into account in Desvillettes and Lorenzani, ${ }^{37}$ about the accuracy of the BGK approximation we consider valid the same remarks reported in that reference for a single component gas (still lacking a complete experimental data set).

\section{PRELIMINARY HYDRODYNAMIC CONSIDERATIONS}

In the low-frequency limit, the problem concerning the propagation of sound waves of small amplitudes in a binary gas mixture is correctly described by the classical Navier-Stokes, Fourier, 
and Fick equations. ${ }^{59,60}$ As the sound frequency increases, the usual Navier-Stokes description becomes unable to describe the experimental data and, in particular, leads to an infinite phase velocity as the frequency goes to infinity. The analysis of the gas-mixture equations is more difficult than the comparable single component gas theory because of the many different scales which now enter in the approach to equilibrium. There is the approach of the distribution function to a Maxwellian distribution (referred to as Maxwellization) and, in addition, there is the equilibration of the species (i.e., the vanishing of differences in velocity and temperature among the species). Some time ago, Grad conjectured that when a binary gas mixture is made up of species with very different molecular masses, the approach of the gas to equilibrium should exhibit very different relaxation times. ${ }^{18}$ First, the light species and then the heavy species reach approximate local Maxwell equilibrium about independent species temperatures, in times of the orders of the different species self-collision times: $\theta_{1}$ for the light-species and $\theta_{2}$ for the heavy species. Equilibration between the species can only be attained thereafter on a time scale of order $\theta_{\Delta T}$, which is a relaxation time for the difference in temperature $(\Delta T)$ between the species. When one assumes comparable number densities $n^{1} \simeq n^{2}$, the times characterizing these different epochs are expected to stand in the ratios, ${ }^{21,35}$

$$
\frac{\theta_{1}}{\theta_{2}} \simeq \frac{\theta_{2}}{\theta_{\Delta T}} \simeq\left(\frac{m^{1}}{m^{2}}\right)^{1 / 2}
$$

with $m^{1}$ and $m^{2}$ being the molecular masses of the light and heavy species, respectively. If $\left(\mathrm{m}^{1} / \mathrm{m}^{2}\right)$ is very small, as it happens for disparate-mass gas mixtures, then $\theta_{\Delta T}$ becomes of the order of a typical macroscopic time and $\Delta T$ is permitted to be a large effect. For completeness, a more detailed study of all the parameters which play a role in the approach to the equilibrium of different gaseous mixtures, starting from the BGK-Boltzmann equations considered in the present paper, has been reported in Appendix B.

Correct equations which necessarily involve separate species temperatures in part of the continuum regime have been developed independently by several authors. Goebel et al $^{21}$ derived a two-temperature hydrodynamic description by using Grad's thirteen moment approach for a binary mixture of Maxwell molecules. The regime of interest here is the frequency regime $\omega \simeq \theta_{\Delta T}^{-1}$. Using a linearized version of the equations obtained by Goebel et al. ${ }^{21}$ Huck and Johnson ${ }^{30}$ suggested the possibility of several simultaneous sound modes in a disparate-mass gas mixture, for a narrow range of compositions at a high enough frequency. Sound propagation experiments were carried out on $\mathrm{He}-\mathrm{Xe}$ mixtures, in the two-temperature regime, by Bowler. ${ }^{32-34}$ The most significant aspect highlighted in the Bowler experimental data is that there is, indeed, a large-scale change in dispersion at high frequencies as the He mole fraction $n_{0}^{1}$ goes from 0.2 to 0.8 . This change occurs at roughly $n_{0}^{1} \simeq 0.5$ for frequencies above $v / p \simeq 70 \mathrm{MHz} / \mathrm{atm}$. The dispersion observed by Bowler confirms the predictions of Huck and Johnson in showing clear evidence of a competition between two sound modes excited in disparate-mass gas mixtures, and in verifying the predicted values for critical composition and frequency which should characterize this interference. Referring to the results found by Huck and Johnson, one can conclude that for frequencies above the critical frequency there can be a slow wave or a fast wave in the gas, with the possibility that both may be present simultaneously if $\mathrm{He}$ and $\mathrm{Xe}$ are present in nearly equal proportions. The slow wave itself is a characteristic of a Xe-rich mixture, while the fast wave is the characteristic of the one in which $\mathrm{He}$ predominates. Closer study of the phase and amplitude for the deviation of the hydrodynamic variables from equilibrium confirms that the slow wave is a damped soundlike mode primarily carried by the heaviest component of the mixture, which is partly decoupled from the light component, but the precise identity of these interfering modes has never been completely understood over the years.

\section{RESULTS AND DISCUSSION}

The results presented here refer to the noble gaseous mixtures of $\mathrm{He}-\mathrm{Xe}$ (that is, helium with molecular mass $m^{1}=4.0026$ au and xenon with molecular mass $m^{2}=131.29$ au) and $\mathrm{Ne}-\mathrm{Ar}$ (that is, neon with molecular mass $m^{1}=20.179$ au and argon with molecular mass $m^{2}=39.948 \mathrm{au}$ ). The computations have been carried out for three different values of the rarefaction parameter $\delta=$ $0.1,1,10$ and of the ratio of the molar concentrations of the two species $N_{12}=n_{0}^{1} / n_{0}^{2}=0.11,1,9$ at 


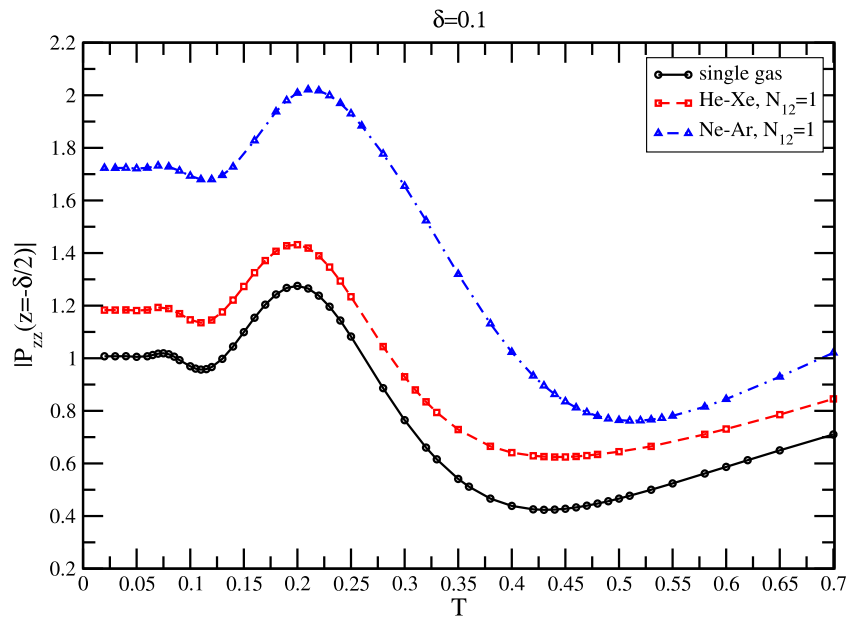

FIG. 1. Amplitude of the normal stress tensor $P_{z z}$ at the oscillating wall versus $T$ for $\delta=0.1$. Comparison between the results obtained for a single component gas (circles) and for the $\mathrm{He}-\mathrm{Xe}$ (squares) and $\mathrm{Ne}-\mathrm{Ar}$ (triangles) mixtures, with the same molar concentrations.

varying the period $T$ of oscillation of the moving plate. For a single component gas, it has been pointed out by Desvillettes and Lorenzani ${ }^{37}$ that above a certain frequency of oscillation of the lower wall of the channel, the sound waves propagating through the gas are trapped in the gaps between the moving elements and the fixed boundaries of the microdevice. In particular, Desvillettes and Lorenzani $^{37}$ found a scaling law (valid for all Knudsen numbers) that predicts a resonant response of the system when the dimensionless distance between the channel walls (measured in units of the oscillation period of the moving plate), $L=\frac{\delta}{T}$, takes a well-defined fixed value. A resonant/antiresonant response of the system can be detected due to the constructive/destructive interference occurring between the incident and reflected sound waves generated by the high-frequency oscillating boundary of the microchannel. Corresponding to a resonant response of the system, the amplitude of $P_{z z}$ at $z=-\delta / 2$ reaches its maximum value (resonance) or its minimum value (antiresonance). In particular, the occurrence of an antiresonance is of paramount importance, since if the device is operated close to the corresponding frequency, the damping due to the gas is considerably reduced.

In Figures 1-3, we report the profiles of the global normal stress amplitude at the oscillating wall, obtained by numerical integration of Eqs. (24)-(27), as a function of the period $T$, for three

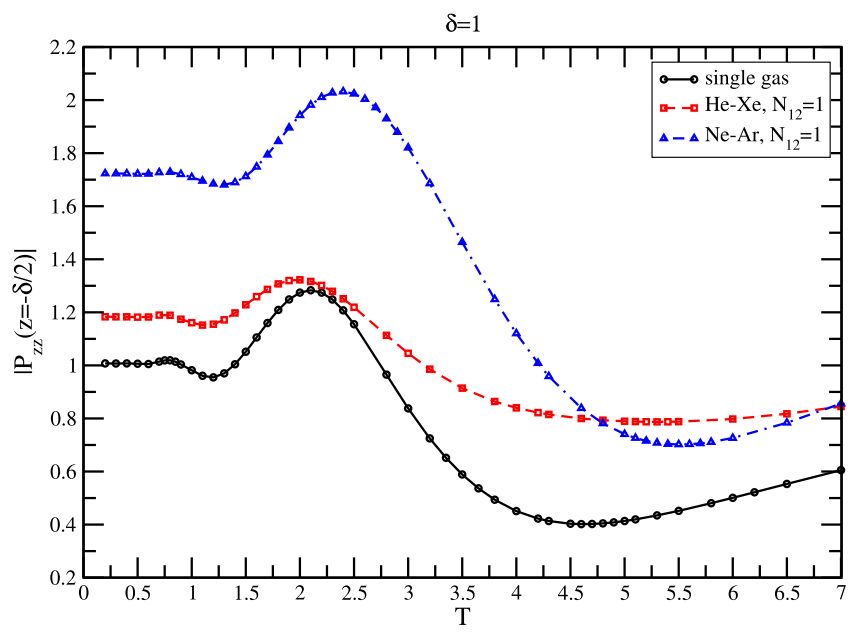

FIG. 2. Amplitude of the normal stress tensor $P_{z z}$ at the oscillating wall versus $T$ for $\delta=1$. Comparison between the results obtained for a single component gas (circles) and for the $\mathrm{He}-\mathrm{Xe}$ (squares) and $\mathrm{Ne}-\mathrm{Ar}$ (triangles) mixtures, with the same molar concentrations. 


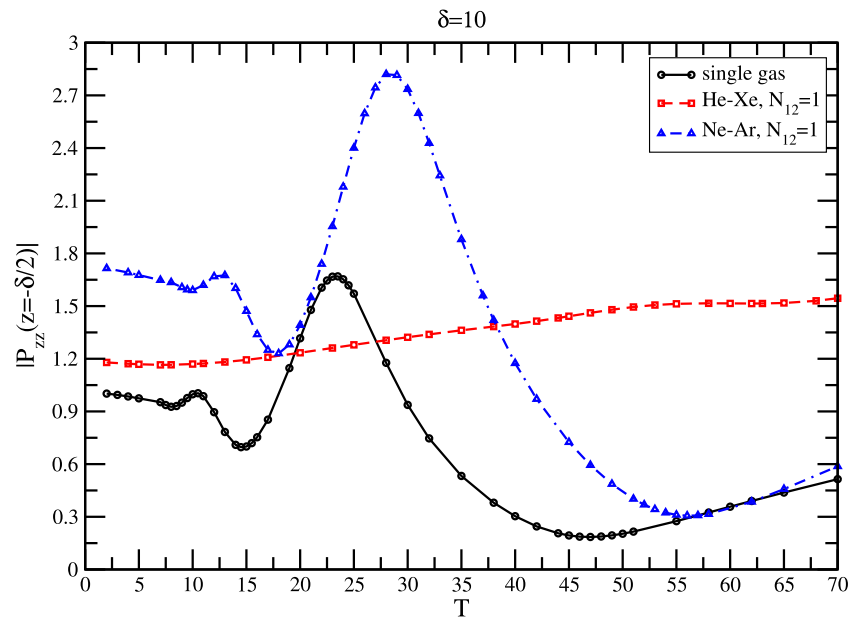

FIG. 3. Amplitude of the normal stress tensor $P_{z z}$ at the oscillating wall versus $T$ for $\delta=10$. Comparison between the results obtained for a single component gas (circles) and for the $\mathrm{He}-\mathrm{Xe}$ (squares) and $\mathrm{Ne}-\mathrm{Ar}$ (triangles) mixtures, with the same molar concentrations.

different values of the rarefaction parameter $\delta$. We have included in these pictures the results of numerical calculations for the mixtures $\mathrm{He}-\mathrm{Xe}, \mathrm{Ne}-\mathrm{Ar}$ (with the same molar concentrations, $\left.N_{12}=1\right)$, as well as those corresponding to a single component gas. Figures 1-3 reveal that, in the near-free molecular flow regime $(\delta=0.1)$ and in the transitional region $(\delta=1)$, the resonant response of the system occurs also for both gas mixtures considered (although the scaling law found for a single component gas does not hold any longer), while, in the near-continuum regime $(\delta=10)$, the propagation of sound waves in the disparate-mass He-Xe mixture does not show up any resonance.

For completeness, we list in Table I the values of $T$ corresponding to the occurrence of resonances $\left(T_{r}\right)$ and antiresonances $\left(T_{a}\right)$, in the case of a single component gas and of both mixtures

TABLE I. Values of $T$ corresponding to the occurrence of resonances $\left(T_{r}\right)$ and antiresonances $\left(T_{a}\right)$, for a single component gas and for the mixtures $\mathrm{Ne}-\mathrm{Ar}$ and $\mathrm{He}-\mathrm{Xe}$, for different values of $N_{12}$ and $\delta$. The symbol "--" indicates a lack of resonances/antiresonances.

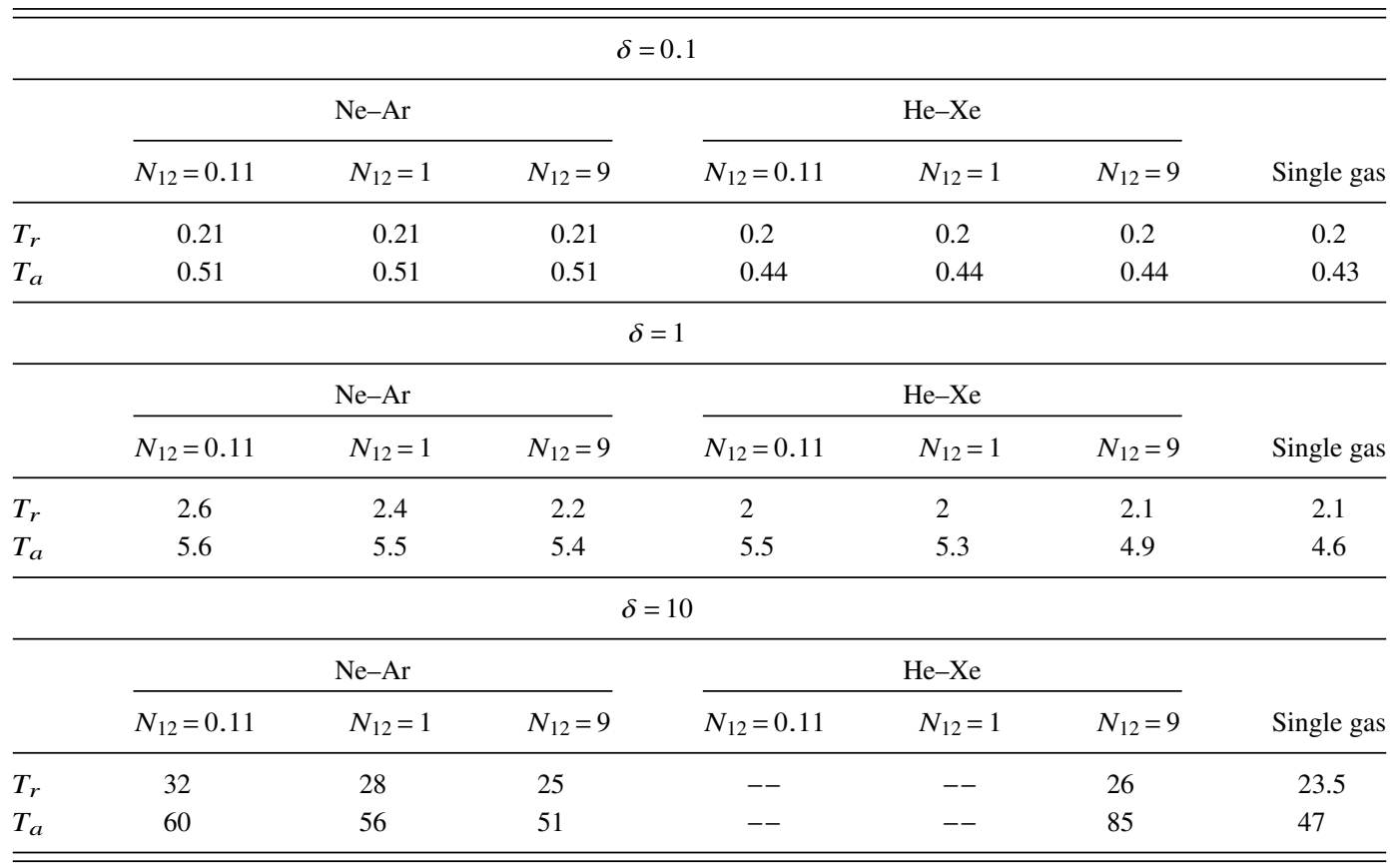


$\mathrm{Ne}-\mathrm{Ar}$ and $\mathrm{He}-\mathrm{Xe}$, for different values of the ratio of the molar concentrations of the two species $N_{12}$ and of the rarefaction parameter $\delta$. In order to inspect more deeply the physical processes going on in both gas mixtures, we examine in the following the macroscopic profiles of the mixture components in different regimes.

\section{A. Near-continuum regime}

We report in Figures 4-5 the velocity and temperature profile of the mixture components as a function of the distance across the gap of the channel (at different stages during a period of oscillation). We have included in these pictures the results of numerical calculations for the mixtures $\mathrm{Ne}-\mathrm{Ar}$ and $\mathrm{He}-\mathrm{Xe}$ (with $N_{12}=1$ ), when the period $T$ matches the main resonant response of the system and $\delta=10$. Since for the He-Xe mixture no resonances appear, we have chosen a period $T$ corresponding to the occurrence of the main resonance for a single component gas. In all the figures presented in the following, the lightest component of each mixture has been labelled with superscript 1 , while the heaviest one with superscript 2 . For a single gas, it has been pointed out by Desvillettes and Lorenzani ${ }^{37}$ that, when a resonant response of the system occurs, the velocity field of the gas takes the form of a standing wave and concomitantly also the temperature field assumes a characteristic shape. In the case of the $\mathrm{Ne}-\mathrm{Ar}$ mixture, both species have the same macroscopic fields (see Fig. 4). In particular, the velocity of each species takes the form of a standing wave, as it happens for a single gas when a resonant response of the system occurs. On the contrary, for the He-Xe mixture, the pictures reveal that two different forced-sound modes are simultaneously present: a fast and a slow wave (see Fig. 5). The slow wave is a damped soundlike mode primarily carried by the Xe, while the fast wave should be associated to the He-component of the mixture. These observations are in agreement with previous predictions about the possibility of several simultaneous sound modes in a disparate-mass gas mixture for a narrow range of compositions at high enough frequencies. ${ }^{30,33,35}$ In particular, the analysis performed by Huck and Johnson ${ }^{30}$ (briefly

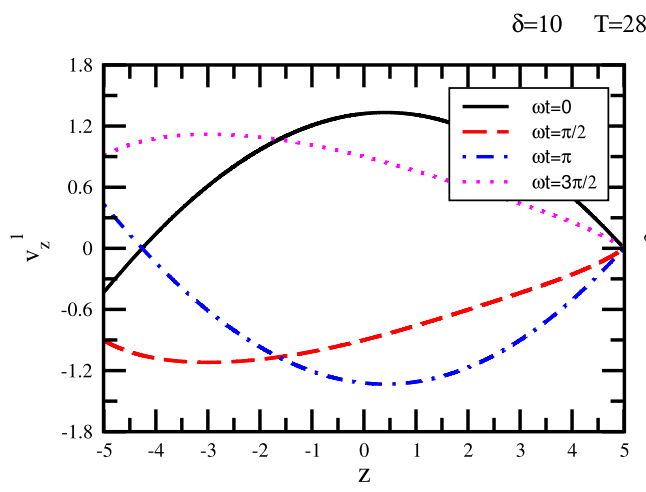

$\mathrm{N}_{12}=1 \quad(\mathrm{Ne}-\mathrm{Ar})$
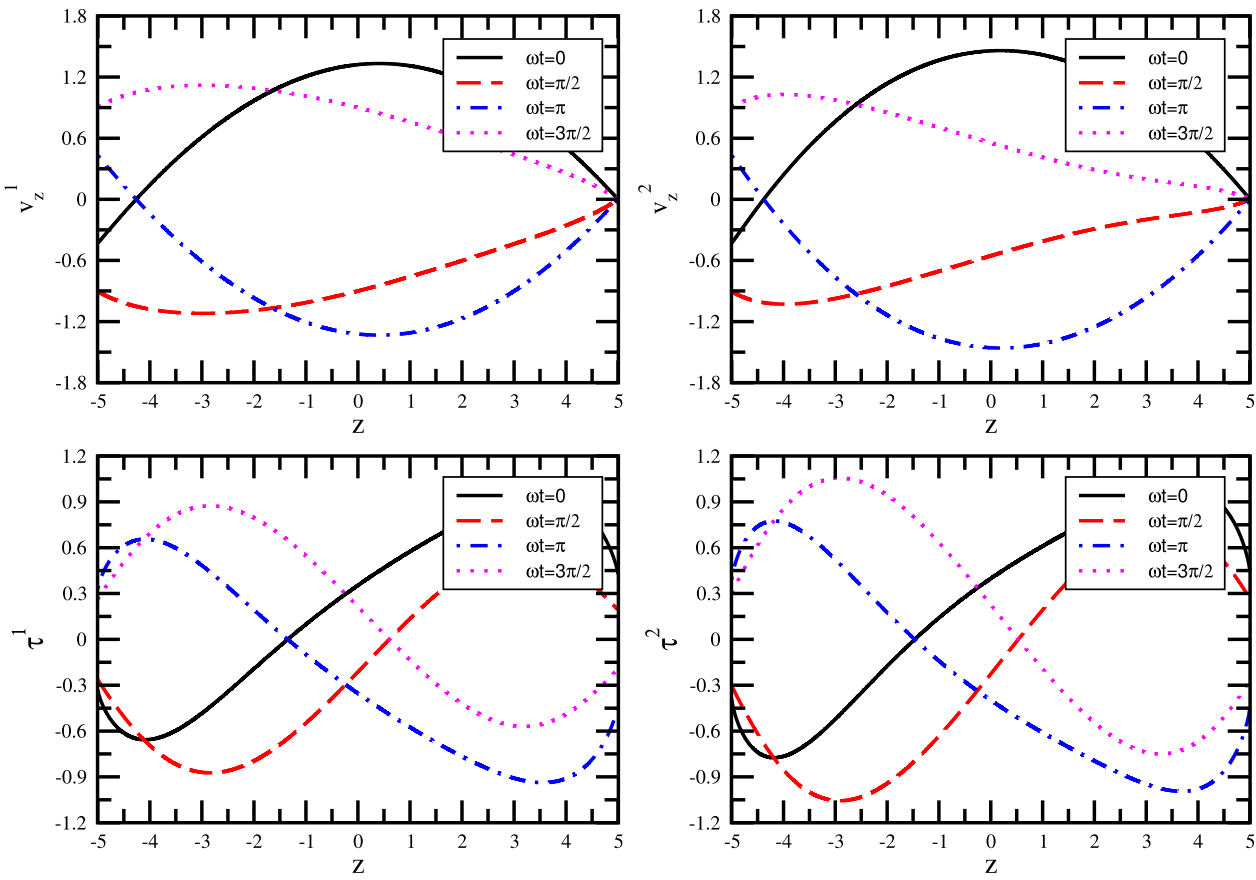

FIG. 4. Variation of the macroscopic velocities and temperatures of the Ne-Ar mixture components (with the same molar concentrations), in the $z$-direction across the gap of the channel for $\delta=10$ and $T=28$. In each panel, the profiles of the macroscopic quantities are shown at different stages during a period of oscillation of the moving wall. 


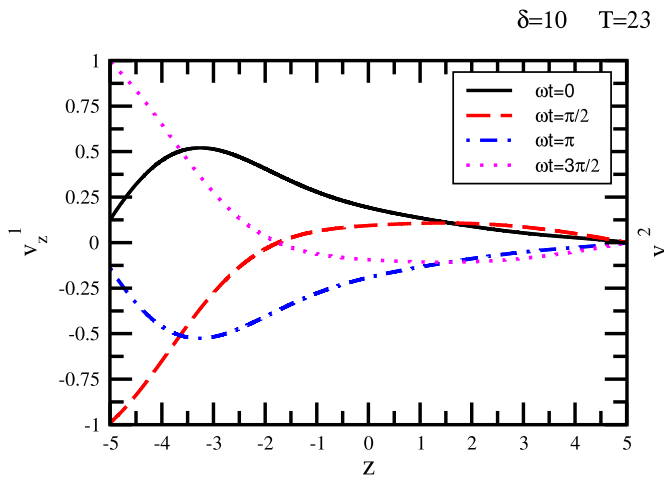

$\mathrm{N}_{12}=1 \quad(\mathrm{He}-\mathrm{Xe})$
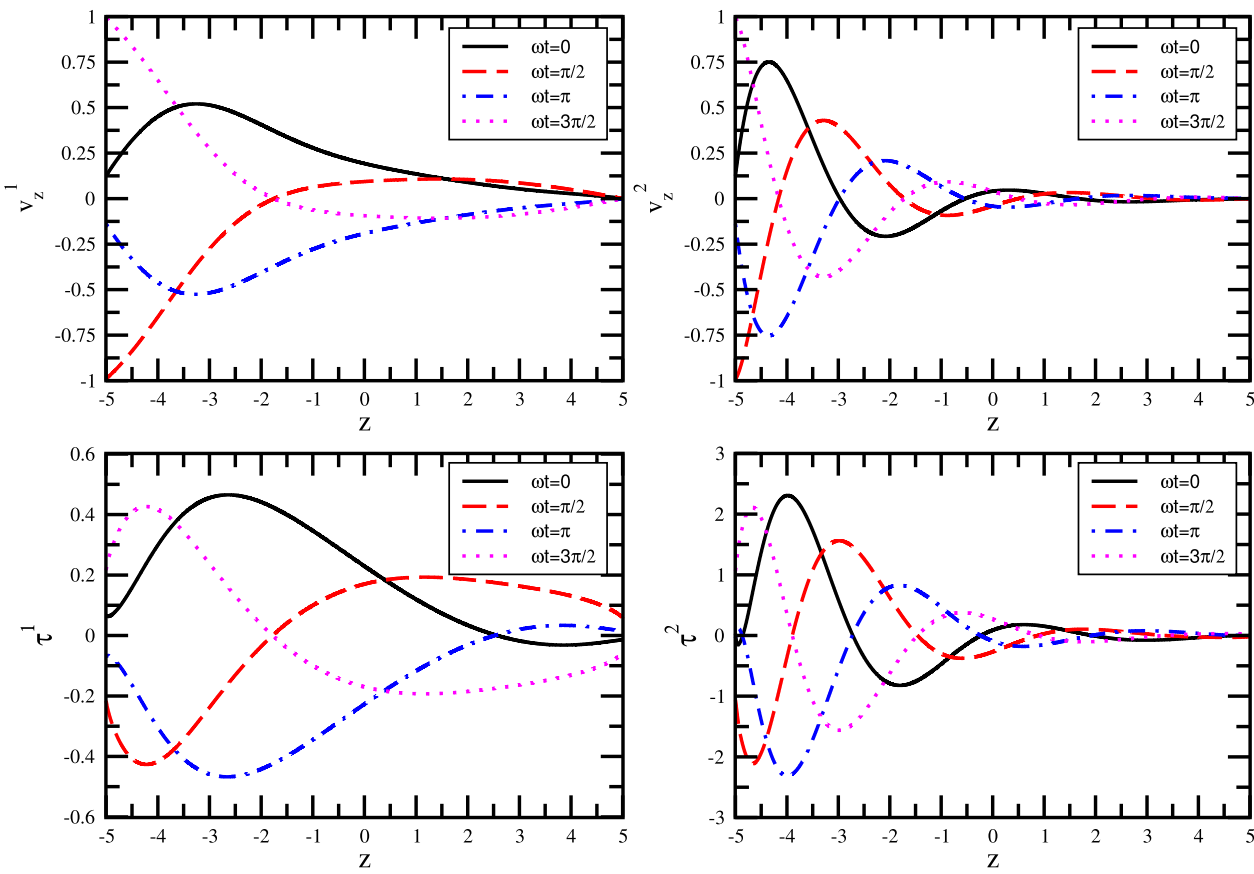

FIG. 5. Variation of the macroscopic velocities and temperatures of the He-Xe mixture components (with the same molar concentrations), in the $z$-direction across the gap of the channel for $\delta=10$ and $T=23$. In each panel, the profiles of the macroscopic quantities are shown at different stages during a period of oscillation of the moving wall.

reported in Section IV), which predicted the existence of two sound modes of comparable absorption but very different speed of propagation, seems confirmed by our numerical results. Indeed, looking at Figures 6 and 7, where the velocity and temperature profiles of the mixture components are shown for different values of the period $T(T=2$ and $T=70)$, one can infer that the characteristics of the macroscopic fields, associated with the two sound modes, depend strongly on the excitation frequency of the modes themselves. At $T=2$, both modes are damped, then, when $T$ increases, they tend to equilibrate, even if, in a neighborhood of $T=23$, the sound mode associated to the heaviest component of the mixture appears more damped than the other and with a very different speed of propagation. Since this trend is also confirmed for $N_{12}=9$ and $N_{12}=0.11$, we can conclude that the equilibration of the modes associated with the mixture components is not related to the number of cross collisions between the molecules of the two species, as one might expect. ${ }^{18,35}$ Therefore, the results obtained previously, based largely on the two-temperature hydrodynamic description and summarized in Section IV, provide only a partial picture of the real characteristics of the macroscopic fields associated with different sound modes propagating in a disparate-mass gas mixture.

To explain the lack of resonances in a disparate-mass gas mixture, when $N_{12}=1$, we can resort to a qualitative analysis of the integral equations given by Eqs. (47) and (48). In the near-continuum regime $(\delta \geq 10)$, the integrals in Eqs. (47) and (48) give the main contribution to the velocities of gas components. In spite of the complex form assumed by these expressions, some qualitative properties on the behaviour of the two species of the mixture can still be deduced. For mixtures like Ne-Ar, whose constituents have comparable molecular mass, such that $\gamma \simeq \tilde{\gamma}, \Theta_{12} \simeq 1$, and $M_{12} \simeq 1$, the velocity profiles of the two species are symmetric by interchanging superscripts 1 and 2 (as the numerical results reported in Fig. 4 show). In this case, Eq. (47) reduces to the equation for the velocity field of a single-component gas, ${ }^{37}$ since the terms $\left(-\Gamma^{1} v_{z}^{1}\right)$ and $\left(\Gamma^{1} v_{z}^{2}\right)$ cancel out as well as the terms: $-\left[\frac{2 \Gamma^{1} M_{12}}{\left(1+M_{12}\right)}\right] \tau^{1}$ and $\left[\frac{2 \Gamma^{1} M_{12}}{\left(1+M_{12}\right)}\right] \tau^{2}$. All the remarks done by Desvillettes and Lorenzani ${ }^{37}$ on the appearance of resonances/antiresonances will continue to apply, except that now, 


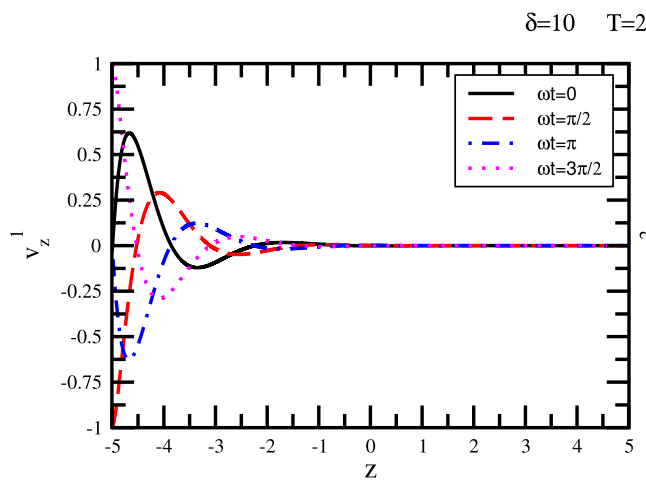

$\mathrm{N}_{12}=1 \quad(\mathrm{He}-\mathrm{Xe})$
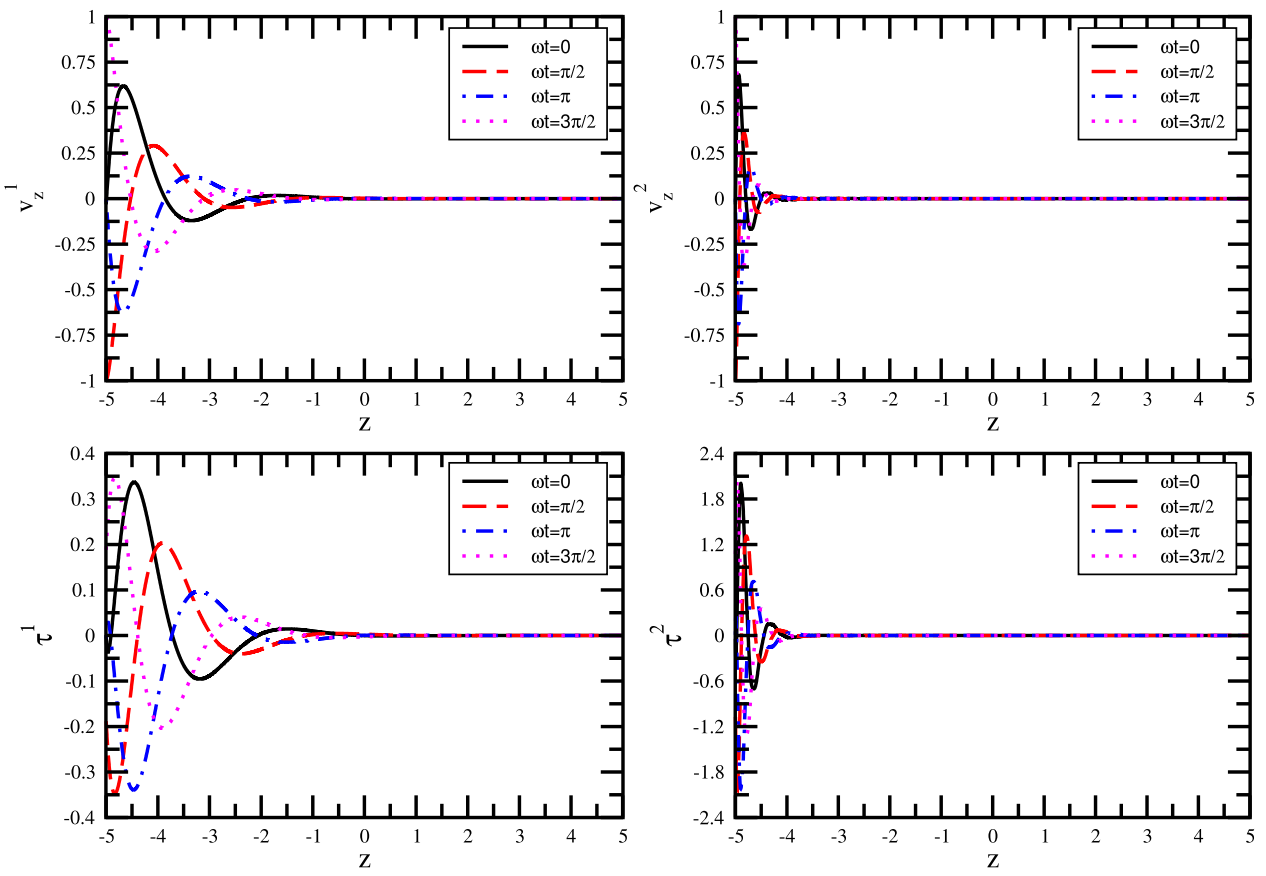

FIG. 6. Variation of the macroscopic velocities and temperatures of the He-Xe mixture components (with the same molar concentrations), in the $z$-direction across the gap of the channel for $\delta=10$ and $T=2$. In each panel, the profiles of the macroscopic quantities are shown at different stages during a period of oscillation of the moving wall.

for a mixture, the phenomenon of constructive/destructive interference will involve incident and reflected waves associated to two species (since the profiles of $\mathrm{v}_{z}^{1}$ and $\mathrm{v}_{z}^{2}$ are symmetric). Therefore, the location of the main resonance (antiresonance) will be different from that found for a single gas (see Figs. 1-3 and Table I).

On the contrary, for mixtures like He-Xe, whose constituents have very different molecular mass, such that $\Theta_{12} \ll 1, M_{12} \ll 1$, and the real and imaginary parts of $\tilde{\gamma}$ are higher than those of $\gamma$, the field $\mathrm{v}_{z}^{2}$, described only by the Abramowitz functions which depend on $\tilde{\gamma}$, approaches zero more quickly, as function of $z$, than $\mathrm{v}_{z}^{1}$. This is true for each value of $T$. Therefore, in this case, the appearance of resonances/antiresonances should be linked only to the constructive/destructive interference occurring between the incident and reflected sound waves associated to the lightest species (He). Indeed, Fig. 3 shows that, for $\delta=10$ and $N_{12}=1$, the propagation of sound waves in the He-Xe mixture does not show up any resonance. In order to trace back the origin of this phenomenon, we are led to compare Eq. (47), when $M_{12} \simeq 0$, with the integral equation for the macroscopic velocity field of a single-component gas, reported in Desvillettes and Lorenzani ${ }^{37}$ (Eq. (67)). The comparison reveals that if we consider values of $T$ in a neighborhood of $T=23$ (corresponding to the main resonance of the system for a single component gas) such that $v_{z}^{2}$ is a rapidly damped profile (see Fig. 5), the velocity field of the lightest species $\mathrm{v}_{z}^{1}$ is the same as that reported by Desvillettes and Lorenzani ${ }^{37}$ for a single gas, except that, in Eq. (47), the influence of the integral term involving $\mathrm{v}_{z}^{1}$ is weighted by the factor $\left(1-\Gamma^{1}\right)$ instead of 1 . This means that the wave associated to the He-component is not a single gas-based sound mode. When $\Gamma^{1} \rightarrow 0$, that is, for $N_{12} \gg 1$, Eq. (47) reduces to the equation for the velocity field of a single-component gas and the resonant phenomenon can be observed, in the near-continuum regime, also for a disparate-mass gas mixture. This is shown in Fig. 8, where we report the profile of the global normal stress amplitude at the oscillating wall as a function of the period $T$, for the He-Xe mixture, when $N_{12}=9$.

By definition, $\Gamma^{1}$ is proportional to the ratio between the microscopic collision frequency between particles of species 1 and $2, v_{1}^{12}$, and the macroscopic collision frequency $v_{1}$. Therefore, one 


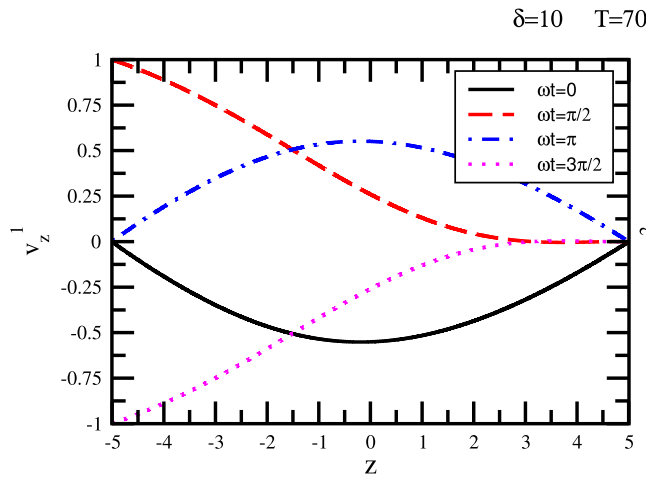

$\mathrm{N}_{12}=1 \quad(\mathrm{He}-\mathrm{Xe})$
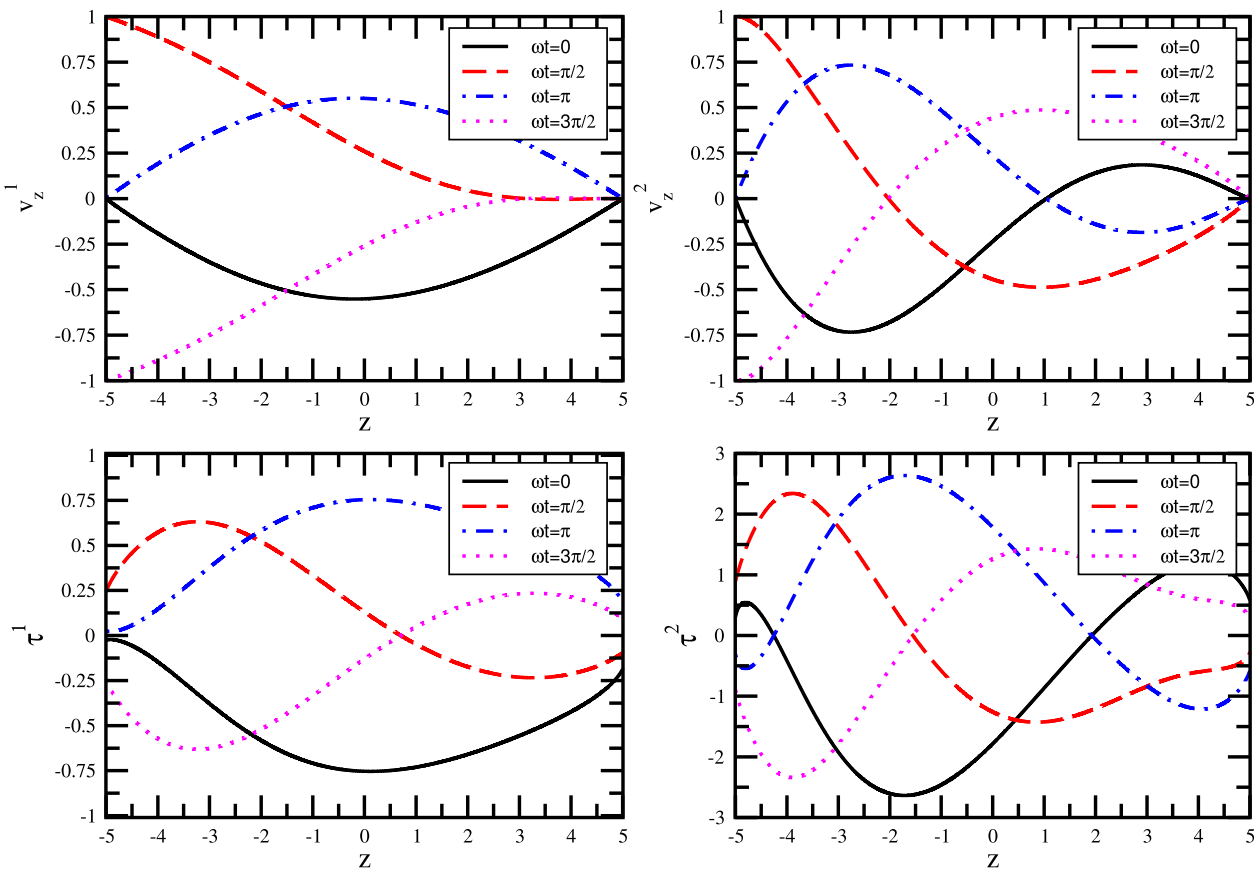

FIG. 7. Variation of the macroscopic velocities and temperatures of the He-Xe mixture components (with the same molar concentrations), in the $z$-direction across the gap of the channel for $\delta=10$ and $T=70$. In each panel, the profiles of the macroscopic quantities are shown at different stages during a period of oscillation of the moving wall.

can conclude that the lack of resonances for a disparate-mass gas mixture is due to the fact that, in the near-continuum regime, when there is a large number of collisions between the two species if their molar concentration is nearly the same $\left(N_{12}=1\right)$, the particles of the heaviest component of the mixture slow down those of the lightest species and no sound waves can reach the fixed wall of the channel. On the contrary, when $\Gamma_{1}$ is small, which can be obtained if $n_{0}^{1} \gg n_{0}^{2}$ (that is, $N_{12} \gg 1$ ), the two species behave almost independently of each other, as it happens in the near free-molecular flow regime, so that the sound waves associated to the He-component of the mixture can again interfere to produce resonances. Therefore, in contrast to that previously obtained by

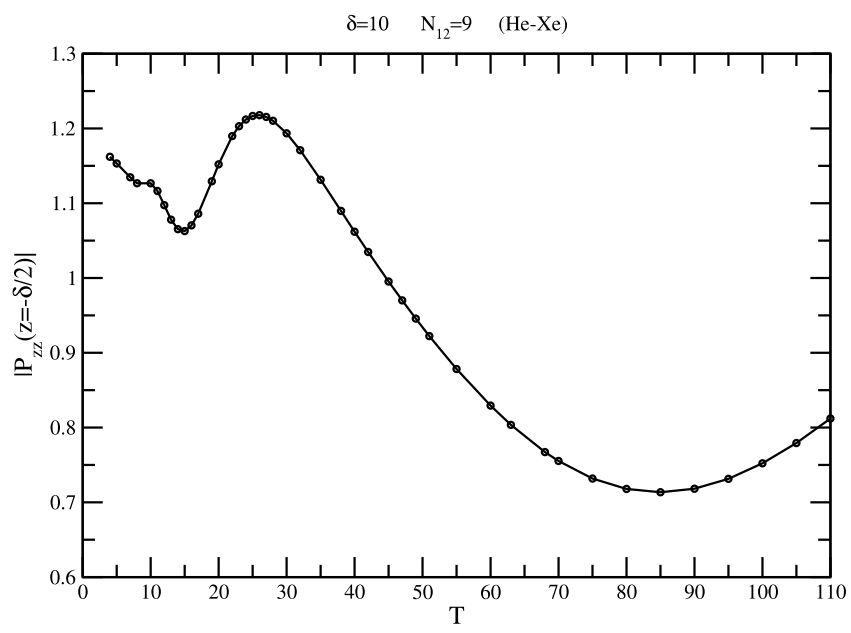

FIG. 8. Amplitude of the normal stress tensor $P_{z z}$ of the He-Xe mixture at the oscillating wall versus $T$ for $\delta=10$ and $N_{12}=n_{0}^{1} / n_{0}^{2}=9$. 
Huck and Johnson, ${ }^{30}$ our results reveal that, in a disparate-mass gas mixture, two excited sound modes always coexist, regardless of the molar concentration of the two species (that is, for each $N_{12}$ ). On the other hand, in agreement with what previously found both theoretically and experimentally, our computations predict a change in the propagation properties of the two excited sound modes when $N_{12}$ ranges from 0.11 to 9 . This can be immediately inferred by comparing the profile corresponding to $\mathrm{He}-\mathrm{Xe}$ shown in Fig. 3 (which has a similar shape also for $N_{12}=0.11$ ) with the one in Fig. 8.

\section{B. Near-free molecular flow regime and transitional region}

In the near-free molecular flow regime $(\delta=0.1)$, the macroscopic field profiles of the lightest species of both mixtures coincide with those obtained for a single gas (see Figures 9-10). This justifies the appearance of resonances/antiresonances for both the mixtures considered. In the case of the Ne-Ar mixture, when the period $T$ matches the main resonant response of the system, the velocity and temperature profiles of the two gas components are similar in shape, even if quantitatively different (see Fig. 9). In particular, the temperature profiles of the two species show a different functional form, reflecting the fact that the "two-temperature regime" is not a prerogative of disparate-mass gas mixtures. In the case of the He-Xe mixture, Fig. 10 reveals that, for $\delta=0.1$, when the period $T$ matches the main resonant response of the system, two different forced-sound modes are simultaneously present: a fast and a slow wave, with the same properties already analyzed in Subsection V A. The only difference with the near-continuum case is that, for $\delta=0.1$, the wave associated to the He-component of the mixture appears to be a single component gas-based sound mode for each value of $N_{12}$, since the velocity field $\mathrm{v}_{z}^{1}$ for helium is the same as that obtained for a simple monatomic gas. The output of the numerical simulations presented in Figures 9-10 can be more deeply evaluated through an analysis of the integral equations given by Eqs. (47) and (48). In the near-free molecular flow regime, the integrals appearing in Eqs. (47) and (48) vanish and the velocity profiles of both components of the mixture are given by the same
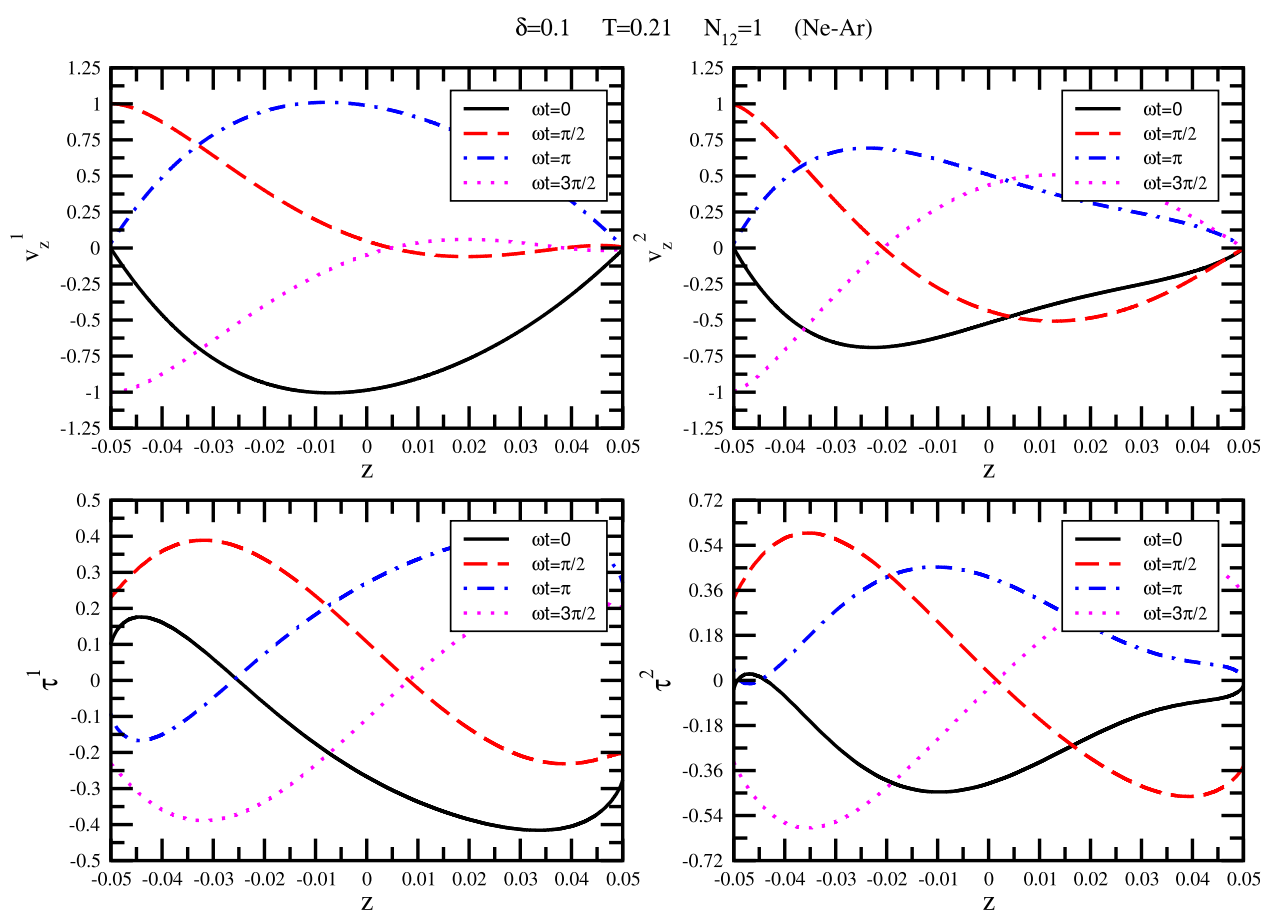

FIG. 9. Variation of the macroscopic velocities and temperatures of the Ne-Ar mixture components (with the same molar concentrations), in the $z$-direction across the gap of the channel for $\delta=0.1$ and $T=0.21$. In each panel, the profiles of the macroscopic quantities are shown at different stages during a period of oscillation of the moving wall. 


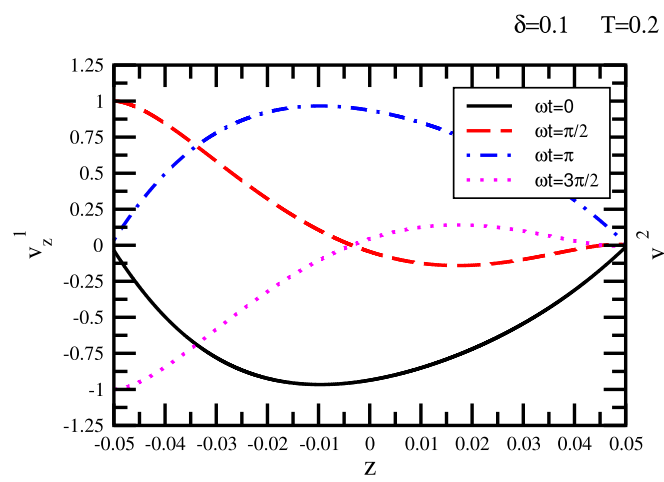

$\mathrm{N}_{12}=1 \quad(\mathrm{He}-\mathrm{Xe})$
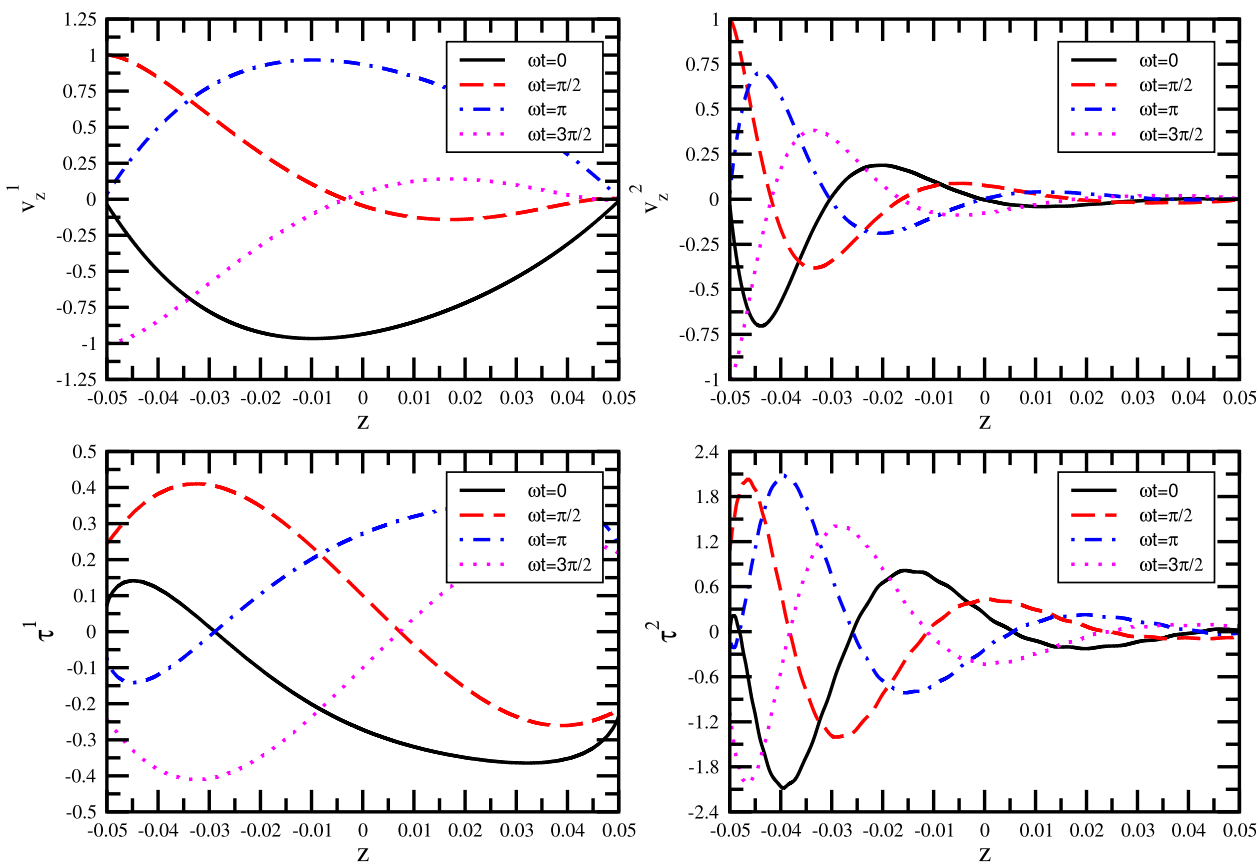

FIG. 10. Variation of the macroscopic velocities and temperatures of the He-Xe mixture components (with the same molar concentrations), in the $z$-direction across the gap of the channel for $\delta=0.1$ and $T=0.2$. In each panel, the profiles of the macroscopic quantities are shown at different stages during a period of oscillation of the moving wall.

expression except that the Abramowitz functions depend on $\gamma$, in the case of $\mathrm{v}_{z}^{1}$, and on $\tilde{\gamma}$, in the case of $\mathrm{v}_{z}^{2}$. Therefore, for mixtures like $\mathrm{Ne}-\mathrm{Ar}$, whose constituents have comparable molecular mass, the parameters $\gamma$ and $\tilde{\gamma}$ take close values and the macroscopic velocity fields $v_{z}^{1}$ and $v_{z}^{2}$ have a similar shape (as shown in Fig. 9). On the contrary, for disparate-mass gas mixtures like $\mathrm{He}-\mathrm{Xe}$, the Abramowitz functions, which depend on $\tilde{\gamma}$, approach zero more quickly as functions of $z$ than those depending on $\gamma$, and the field $\mathrm{v}_{z}^{2}$ represents a damped soundlike mode associated to the heaviest component of the mixture (as shown in Fig. 10). However, also in this case, the same remarks valid for $\delta=10$ can be applied: when the period $T$ is very small (of the order of $10^{-2}$ ), both modes associated to the two mixture components are damped, while, when $T$ increases, they tend to equilibrate. This result confirms that the equilibration of the modes associated with the two species is not related to the number of collisions between molecules, which, in the near free-molecular flow regime, is small. The same considerations hold also for $N_{12}=0.11$ and $N_{12}=9$ and for each $\delta$ lying in the transitional region (that is, $0.1<\delta<10$ ).

\section{CONCLUDING REMARKS}

In the present paper, we have analyzed the high-frequency sound wave propagation in binary mixtures flowing through microchannels, within the framework of kinetic theory of rarefied gases. The results presented refer to the noble gaseous mixtures of $\mathrm{He}-\mathrm{Xe}$ and $\mathrm{Ne}-\mathrm{Ar}$ with different molar concentrations. Our analysis shows that, due to the constructive/destructive interference occurring between the incident and reflected sound waves generated by the oscillating boundary of the microdevice, a resonant/antiresonant response of the system can be detected in the near-free molecular flow regime and in the transitional region for both gas mixtures considered. In the near-continuum regime, for mixtures like $\mathrm{Ne}-\mathrm{Ar}$, the macroscopic velocities of the two species are symmetric and reduce to the velocity field of a single-component gas. In this case, resonances still occur but since the phenomenon of constructive/destructive interference will involve incident and reflected waves associated to two species, the location of the main resonance (antiresonance) turns out to 
be different from that found for a single gas. Instead, when $\delta \geq 10$ and $N_{12} \leq 1$, the propagation of sound waves in disparate-mass gas mixtures (like He-Xe) does not show up any resonance. For a period $T$ corresponding to the occurrence of the main resonance for a single component gas, the macroscopic velocity of the heaviest component $\mathrm{Xe}$ is a rapidly damped field while the velocity of the lightest species He differs from that of a single-component gas due to a term proportional to $\Gamma^{1}$. When $\Gamma^{1} \rightarrow 0$, that is, for $N_{12} \gg 1$, the resonant phenomenon can be observed, in the near-continuum regime, also for a disparate-mass gas mixture and the fast wave carried by the $\mathrm{He}$ appears to be a single component gas-based sound mode.

Therefore, the sound wave propagation model considered in the present paper allows us to analyze the precise nature of the multiple forced-sound modes excited in gaseous mixtures, thanks to the peculiar characteristics shown by the sound waves in correspondence with the resonant/antiresonant frequency. In this regard, our findings extend the results obtained previously, based largely on the two-temperature hydrodynamic description, which provide only a partial picture of the real characteristics of the macroscopic fields associated with different sound modes. Despite the fact that the theoretical approaches presented here and in Refs. 30 and 35 assume Maxwellian intermolecular forces, the predictions about the possibility of several simultaneous sound modes in a disparate-mass gas mixture and the physical regimes in which these features should be evident have been all confirmed by the experiments. ${ }^{32-34}$ Therefore, even if the BGK kinetic model, considered in the present paper, has been derived in closed-form by assuming Maxwell molecules, we expect to obtain analogous results on sound waves propagation also for other collision kernels corresponding to more realistic intermolecular potentials.

\section{ACKNOWLEDGMENTS}

The authors are grateful to the GNFM for its financial support.

\section{APPENDIX A: DETAILED FORM OF THE FUNCTIONS APPEARING IN THE INTEGRAL EQUATIONS (47)-(48)}

In the following, we report the explicit form of the functions $\mathcal{K}_{i}(i=1, \ldots, 4)$ appearing in Eqs. (47)-(48):

$$
\begin{gathered}
\mathcal{K}_{1}(z, v):=\frac{2}{\sqrt{\pi}} T_{2}((\delta / 2+z) v)-\frac{1}{\sqrt{\pi}}\left[1-4 T_{1}^{2}(\delta v)\right]^{-1} T_{1}((\delta / 2-z) v) \\
\times\left[2 \sqrt{\pi} T_{1}(\delta v)+4 T_{2}(\delta v)\right]+\frac{1}{\sqrt{\pi}}\left[1-4 T_{1}^{2}(\delta v)\right]^{-1} T_{1}((\delta / 2+z) v) \\
\times\left[\sqrt{\pi}+8 T_{1}(\delta v) T_{2}(\delta v)\right] \\
\mathcal{K}_{2}(z, s, v):=\left[1-4 T_{1}^{2}(\delta v)\right]^{-1} \\
\times\left[4 T_{1}(\delta v) T_{1}((\delta / 2-z) v) T_{0}((\delta / 2+s) v)+2 T_{1}((\delta / 2-z) v) T_{0}((\delta / 2-s) v)\right. \\
\left.-4 T_{1}(\delta v) T_{1}((\delta / 2+z) v) T_{0}((\delta / 2-s) v)-2 T_{1}((\delta / 2+z) v) T_{0}((\delta / 2+s) v)\right] \\
\mathcal{K}_{3}(z, s, v):=\left[1-4 T_{1}^{2}(\delta v)\right]^{-1} \\
\times\left[4 T_{1}((\delta / 2-z) v) T_{1}((\delta / 2-s) v)-8 T_{1}(\delta v) T_{1}((\delta / 2-z) v) T_{1}((\delta / 2+s) v)\right. \\
\left.-8 T_{1}(\delta v) T_{1}((\delta / 2+z) v) T_{1}((\delta / 2-s) v)+4 T_{1}((\delta / 2+z) v) T_{1}((\delta / 2+s) v)\right] \\
\times \mathcal{K}_{4}(z, s, v):=\left[1-4 T_{1}^{2}(\delta v)\right]^{-1} \\
\times\left[T_{1}(\delta v) T_{1}((\delta / 2-z) v)\left[T_{2}((\delta / 2+s) v)-\frac{1}{2} T_{0}((\delta / 2+s) v)\right]+\frac{1}{2} T_{1}((\delta / 2-s) v)\right. \\
\times\left[T_{2}((\delta / 2-s) v)-\frac{1}{2} T_{0}((\delta / 2-s) v)\right]-T_{1}(\delta v) T_{1}((\delta / 2+z) v)\left[T_{2}((\delta / 2-s) v)\right. \\
\left.\left.-\frac{1}{2} T_{0}((\delta / 2-s) v)\right]-\frac{1}{2} T_{1}((\delta / 2+s) v)\left[T_{2}((\delta / 2+s) v)-\frac{1}{2} T_{0}((\delta / 2+s) v)\right]\right]
\end{gathered}
$$




\section{APPENDIX B: PARAMETERS INVOLVED IN THE APPROACH TO EQUILIBRIUM OF DIFFERENT GASEOUS MIXTURES ACCORDING TO THE BGK-BOLTZMANN EQUATION}

Since the collision operator in the BGK model (1) consists of a unique relaxation-type term, the relaxation time which measures the rate of convergence of the distribution function $f^{s}$ towards a Maxwellian distribution is simply given by the inverse of the macroscopic collision frequency: $\theta_{s}=1 / v_{s}$. Therefore, using Eqs. (18) and (19), the times, needed by both mixture components to reach approximate local Maxwell equilibrium about independent species temperatures, are expected to stand in the ratios

$$
\frac{\theta_{1}}{\theta_{2}}=\Theta_{12}=\frac{v_{2}}{v_{1}}=\sqrt{M_{12}} \frac{\left[\sqrt{2 M_{12}} N_{12}+\sqrt{\frac{K^{22}}{K^{12}}} \sqrt{1+M_{12}}\right]}{\left[\sqrt{2}+\sqrt{\frac{K^{11}}{K^{12}}} \sqrt{1+M_{12}} N_{12}\right]} .
$$

If the Chapman-Enskog expressions for the gas viscosity coefficients $\eta^{s}$ are considered (Eq. (5)) and the method of the combination rule taken into account (Eq. (6)), Eq. (B1) reads

$$
\Theta_{12}=\frac{\left[\sqrt{2} M_{12} N_{12}+\sqrt{\frac{\eta^{1}}{\eta^{2}} \sqrt{M_{12}}} \sqrt{1+M_{12}}\right]}{\left[\sqrt{2}+\sqrt{\frac{\eta^{2}}{\eta^{1}} \sqrt{M_{12}}} \sqrt{1+M_{12}} N_{12}\right]} .
$$

For the purpose of obtaining a rough estimate of orders of magnitude, it can be assumed that the species of the mixture have comparable number densities $\left(N_{12} \simeq 1\right)$ and that the intermolecular forces are of the same order $\left(K^{11} \simeq K^{22} \simeq K^{12}\right)$. In this case, Eq. (B1) reduces to

$$
\Theta_{12} \simeq C \sqrt{M_{12}},
$$

where the constant $C$ is of order 1 for all $M_{12} \in[0,1]$ (precisely, $C \in\left[\frac{1}{1+\sqrt{2}}, 1\right]$ ). The estimate (B3) is consistent with the scaling (53) first conjectured by Grad (under the same hypotheses leading to (B3)), even if a complete dependence on all the parameters which play a role in the approach to the equilibrium is given by Eq. (B2). In order to evaluate the relaxation time for the difference in temperature $\theta_{\Delta T}$ (which is the time scale for the equilibration between the species), we have to consider the evolution equation for the difference of species temperatures $\left(T^{1}-T^{2}\right)$. An explicit estimate may be found in physical situations, recently underlined in extended thermodynamics, ${ }^{27}$ when the mechanical relaxation time is shorter than the thermal one, or, in other words, the equalization of species velocities is faster than the equalization of temperatures. In this case, an adequate hydrodynamic description can be obtained by considering a single mass velocity for the gas mixture and different temperatures for each species. Thus, the evolution equation for the temperature $T^{s}$, obtained by multiplying the space homogeneous version of Eq. (1) by $m^{s}|\xi|^{2} /\left(3 k n_{0}^{s}\right)$ and integrating over $\xi$, reads

$$
\frac{\partial T^{s}}{\partial t}=v_{s}\left(T_{s}-T^{s}\right) \quad s=1,2
$$

since we have assumed $\mathbf{v}^{1}=\mathbf{v}^{2}=\mathbf{v}_{1}=\mathbf{v}_{2}=\mathbf{v}_{0}=\mathbf{0}$. Hence, subtracting two Equations (B4), we get

$$
\frac{\partial\left(T^{1}-T^{2}\right)}{\partial t}=v_{1}\left(T_{1}-T^{1}\right)-v_{2}\left(T_{2}-T^{2}\right) \text {. }
$$

Using Eq. (4) and bearing in mind again the assumption $\mathbf{v}^{1}=\mathbf{v}^{2}$, Eq. (B5) becomes

$$
\begin{gathered}
\frac{\partial\left(T^{1}-T^{2}\right)}{\partial t}=-4 \pi A_{1}(5) \sqrt{K^{12}}\left(n^{1}+n^{2}\right) \sqrt{\frac{M_{12}}{m^{2}\left(1+M_{12}\right)^{3}}}\left(T^{1}-T^{2}\right) \\
\equiv-v_{\Delta T}\left(T^{1}-T^{2}\right) .
\end{gathered}
$$


Therefore, from Eqs. (19) and (B6), by assuming that the species of the mixture have comparable number densities $\left(N_{12} \simeq 1\right)$ and that the intermolecular forces are of the same order $\left(K^{11} \simeq K^{22} \simeq\right.$ $K^{12}$ ), we get the following estimate:

$$
\frac{\theta_{2}}{\theta_{\Delta T}}=\frac{v_{\Delta T}}{v_{2}} \simeq \text { const. } \sqrt{M_{12}}
$$

in agreement with Grad expectation (53). ${ }^{35}$

${ }^{1}$ C. S. Wang Chang and G. E. Uhlenbeck, On the Propagation of Sound in Monatomic Gases (Univ. Michigan Engineering Research Institute Project M29, Ann Harbor, 1952).

${ }^{2}$ C. L. Pekeris, Z. Alterman, L. Finkelstein, and K. Frankowski, "Propagation of sound in a gas of rigid spheres," Phys. Fluids 5, 1608 (1962).

${ }^{3}$ M. Greenspan, "Propagation of sound in five monatomic gases," J. Acoust. Soc. Am. 28, 644 (1956).

${ }^{4}$ E. Meyer and G. Sessler, "Schallausbreitung in gasen hohen frequenzen," Z. Physik 149, 15 (1957).

${ }^{5}$ R. Schotter, "Rarefied gas acoustics in the noble gases," Phys. Fluids 17, 1163 (1974).

${ }^{6}$ C. Cercignani and F. Sernagiotto, "The method of elementary solutions for time dependent problems in linearized kinetic theory," Ann. Phys. 30, 154 (1964).

${ }^{7}$ D. Kahn and D. Mintzer, "Kinetic theory of sound propagation in rarefied gases," Phys. Fluids 8, 1090 (1965).

${ }^{8}$ L. Sirovich and J. K. Thurber, "Propagation of forced sound waves in rarefied gasdynamics," J. Acoust. Soc. Am. 37, 329 (1965).

9 J. K. Buckner and J. H. Ferziger, "Linearized boundary value problem for a gas and sound propagation," Phys. Fluids 9 , 2315 (1966).

${ }^{10}$ S. K. Loyalka and T. C. Cheng, "Sound-wave propagation in a rarefied-gas," Phys. Fluids 22, 830 (1979).

11 J. R. Thomas and C. E. Siewert, "Sound-wave propagation in a rarefied-gas," Transp. Theory Stat. Phys. 8, 219 (1979).

${ }^{12} \mathrm{~K}$. Aoki and C. Cercignani, "A technique for time-dependent boundary value problems in the kinetic theory of gases. Part II. Application to sound propagation,” ZAMP 35, 345 (1984).

${ }^{13}$ N. G. Hadjiconstantinou and A. L. Garcia, "Molecular simulation of sound wave propagation in simple gases," Phys. Fluids 13, 1040 (2001).

${ }^{14}$ N. G. Hadjiconstantinou, "Sound wave propagation in a transition-regime micro-and nanochannels," Phys. Fluids 14, 802 (2002).

15 F. Sharipov, W. Marques, and G. M. Kremer, "Free molecular sound propagation,” J. Acoust. Soc. Am. 112, 395 (2002).

${ }^{16}$ R. D. M. Garcia and C. E. Siewert, "The linearized Boltzmann equation: Sound-wave propagation in a rarefied gas," ZAMP 57, 94 (2005).

${ }^{17}$ D. Kalempa and F. Sharipov, "Sound propagation through a rarefied gas confined between source and receptor at arbitrary Knudsen number and sound frequency," Phys. Fluids 21, 103601 (2009).

${ }^{18}$ H. Grad, "Theory of rarefied gases," in Rarefied Gas Dynamics, edited by F. M. Devienne (Pergamon, New York, 1960), pp. 100-138.

${ }^{19}$ E. Goldman and L. Sirovich, "Equations for gas mixtures,” Phys. Fluids 10, 1928 (1967).

${ }^{20}$ J. P. Petit and J. S. Darrozes, "New formulation of the equations of motion of an ionized gas in collision dominated regime," J. Méc. 14(4), 745 (1975).

${ }^{21}$ C. J. Goebel, S. M. Harris, and E. A. Johnson, “Two-temperature disparate-mass gas mixtures: A thirteen moment description," Phys. Fluids 19, 627 (1976).

${ }^{22}$ C. J. Goebel, S. M. Harris, and E. A. Johnson, "Near normal behaviour of disparate-mass gas mixtures," in Rarefied Gas Dynamics, edited by J. L. Potter (American Institute of Aeronautics and Astronautics, New York, 1977), pp. 109-122.

${ }^{23}$ J. D. Ramshaw, "Hydrodynamic theory of multicomponent diffusion and thermal-diffusion in multitemperature gas mixtures," J. Non-Equilib. Thermodyn. 18, 121 (1993).

${ }^{24}$ V. Giovangigli, Multicomponent Flow Modeling. Series on Modeling and Simulation in Science, Engineering and Technology (Birkhäuser, Boston, 1999).

${ }^{25}$ M. Bisi, M. Groppi, and G. Spiga, "Kinetic modelling of bimolecular chemical reactions," in Kinetic Methods for Nonconservative and Reacting Systems, Quaderni di Matematica [Mathematics Series] Vol. 16, edited by G. Toscani (Aracne Editrice, Roma, 2005), pp. 1-143.

${ }^{26}$ E. Kustova and E. Nagnibeda, "On a correct description of a multi-temperature dissociating $\mathrm{CO}_{2}$ flow," Chem. Phys. 321 , 293 (2006).

${ }^{27}$ T. Ruggeri and S. Simic, "Average temperature and Maxwellian iteration in multi-temperature mixtures of fluids," Phys. Rev. E 80, 026317 (2009).

${ }^{28}$ G. M. Kremer, An Introduction to the Boltzmann Equation and Transport Processes in Gases (Springer, Berlin, 2010).

${ }^{29}$ M. Bisi, G. Martalò, and G. Spiga, "Multi-temperature Euler hydrodynamics for a reacting gas from a kinetic approach to rarefied mixtures with resonant collisions," Europhys. Lett. 95, 55002 (2011).

${ }^{30}$ R. J. Huck and E. A. Johnson, "Possibility of double sound propagation in disparate-mass gas mixtures," Phys. Rev. Lett. 44, 142 (1980).

${ }^{31}$ R. J. Huck and E. A. Johnson, "Physical properties of double-sound modes in disparate-mass gas mixtures," in Rarefied Gas Dynamics, edited by S. S. Fisher (American Institute of Astronautics and Aeronautics, New York, 1981), pp. $452-463$.

32 J. R. Bowler, "Harmonic modes in binary monatomic gas mixtures," Ph.D. thesis, University of Surrey, United Kingdom, 1984.

33 J. R. Bowler and E. A. Johnson, “Anomalous dispersion in disparate-mass gas mixtures,” Phys. Rev. Lett. 54, 329 (1985). 
${ }^{34}$ J. R. Bowler and E. A. Johnson, "Anomalous sound propagation and mode degeneracy in noble gas mixtures," Proc. R. Soc. Lond. A 408, 79 (1986).

${ }^{35}$ E. A. Johnson, “Two-temperature hydrodynamics and multiple sound modes in disparate-mass gas mixtures," J. Stat. Phys. 57, 647 (1989)

${ }^{36}$ C. Cercignani, A. Frangi, S. Lorenzani, and B. Vigna, "BEM approaches and simplified kinetic models for the analysis of damping in deformable MEMS," Eng. Anal. Boundary Elem. 31, 451 (2007).

${ }^{37}$ L. Desvillettes and S. Lorenzani, "Sound wave resonances in micro-electro-mechanical systems devices vibrating at high frequencies according to the kinetic theory of gases," Phys. Fluids 24, 092001 (2012).

${ }^{38}$ M. Groppi and G. Spiga, "A Bhatnagar-Gross-Krook-type approach for chemically reacting gas mixtures," Phys. Fluids 16, 4273 (2004).

${ }^{39}$ S. Kosuge, H. Mizuno, and K. Aoki, "Numerical investigation on models of the Boltzmann equation for gas mixtures," in Proceedings of Rarefied Gas Dynamics: 25th International Symposium, edited by M. S. Ivanov and A. K. Rebrov (Siberian Branch of the Russian Academy of Sciences, Novosibirsk, 2007), pp. 286-291.

${ }^{40}$ S. Kosuge, K. Aoki, T. Inoue, D. B. Goldstein, and P. L. Varghese, "Unsteady flows in Io's atmosphere caused by condensation and sublimation during and after eclipse: Numerical study based on a model Boltzmann equation," Icarus 221, 658 (2012).

41 A. Bonucci and S. Lorenzani, "Scaling laws for damping forces exerted by different gases in the near-free molecular flow regimes," Micro and Nanosystems 5, 303 (2013).

${ }^{42}$ S. Chapman and T. G. Cowling, The Mathematical Theory of Non-Uniform Gases (Cambridge University Press, 1970).

${ }^{43}$ C. Cercignani, The Boltzmann Equation and its Applications (Springer, New York, 1988).

${ }^{44}$ F. J. McCormack, "Construction of linearized kinetic models for gaseous mixtures and molecular gases," Phys. Fluids 16, 2095 (1973).

${ }^{45}$ S. Brull, V. Pavan, and J. Schneider, "Derivation of BGK models for mixtures," Eur. J. Mech. (B-Fluids) 33, 74 (2012).

${ }^{46}$ V. Garzó, A. Santos, and J. J. Brey, "A kinetic model for a multicomponent gas," Phys. Fluids A 1, 380 (1989).

${ }^{47}$ G. M. Kremer, M. Pandolfi Bianchi, and A. J. Soares, "A relaxation kinetic model for transport phenomena in a reactive flow," Phys. Fluids 18, 037104 (2006).

${ }^{48}$ F. Sharipov and D. Kalempa, "Velocity slip and temperature jump coefficients for gaseous mixtures.I. Viscous slip coefficient," Phys. Fluids 15, 1800 (2003).

${ }^{49}$ S. Naris, D. Valougeorgis, D. Kalempa, and F. Sharipov, "Gaseous mixture flow between two parallel plates in the whole range of the gas rarefaction," Physica A 336, 294 (2004).

${ }^{50}$ F. Sharipov and D. Kalempa, "Velocity slip and temperature jump coefficients for gaseous mixtures.II. Thermal slip coefficient," Phys. Fluids 16, 759 (2004).

${ }^{51}$ F. Sharipov and D. Kalempa, "Velocity slip and temperature jump coefficients for gaseous mixtures.III. Diffusion slip coefficient," Phys. Fluids 16, 3779 (2004).

52 S. Naris, D. Valougeorgis, D. Kalempa, and F. Sharipov, "Flow of gaseous mixtures through rectangular microchannels driven by pressure, temperature, and concentration gradients," Phys. Fluids 17, 100607 (2005).

${ }^{53}$ P. Andries, K. Aoki, and B. Perthame, "A consistent BGK-type model for gas mixtures," J. Stat. Phys. 106, 993 (2002).

${ }^{54}$ J. Kestin, K. Knierim, E. A. Mason, B. Najafi, S. T. Ro, and M. Waldman, "Equilibrium and transport properties of the noble gases and their mixtures at low density," J. Phys. Chem. Ref. Data 13, 229 (1984).

55 A. B. Huang and D. L. Hartley, "Nonlinear rarefied Couette flow with heat transfer," Phys. Fluids 11, 1321 (1968).

${ }^{56}$ S. Naris and D. Valougeorgis, "Rarefaction effects in the driven cavity flow over the whole range of the Knudsen number," Phys. Fluids 17, 097106 (2005).

${ }^{57} \mathrm{M}$. Bisi and S. Lorenzani, "Damping forces exerted by rarefied gas mixtures in micro-electro-mechanical system devices vibrating at high frequencies," Interfacial Phenomena and Heat Transfer 2(3), 253 (2014).

${ }^{58}$ S. Lorenzani, L. Gibelli, A. Frezzotti, A. Frangi, and C. Cercignani, "Kinetic approach to gas flows in microchannels," Nanoscale Microscale Thermophys. Eng. 11, 211 (2007).

${ }^{59}$ L. D. Landau and E. M. Lifshitz, Fluid Mechanics (Pergamon, New York, 1989).

${ }^{60} \mathrm{~J}$. Lighthill, Waves in Fluids (Cambridge University Press, New York, 1978). 